\title{
An Optimal Control Problem of Forward-Backward Stochastic Volterra Integral Equations with State Constraints
}

\author{
Qingmeng Wei ${ }^{1}$ and Xinling Xiao ${ }^{2}$ \\ ${ }^{1}$ School of Mathematics and Statistics, Northeast Normal University, Changchun 130024, China \\ ${ }^{2}$ School of Mathematics, Shandong University, Jinan 250100, China \\ Correspondence should be addressed to Xinling Xiao; xinlingxiao@mail.sdu.edu.cn
}

Received 26 November 2013; Accepted 2 January 2014; Published 27 February 2014

Academic Editor: Litan Yan

Copyright (c) 2014 Q. Wei and X. Xiao. This is an open access article distributed under the Creative Commons Attribution License, which permits unrestricted use, distribution, and reproduction in any medium, provided the original work is properly cited.

\begin{abstract}
This paper is devoted to the stochastic optimal control problems for systems governed by forward-backward stochastic Volterra integral equations (FBSVIEs, for short) with state constraints. Using Ekeland's variational principle, we obtain one kind of variational inequalities. Then, by dual method, we derive a stochastic maximum principle which gives the necessary conditions for the optimal controls.
\end{abstract}

\section{Introduction}

As we know, with the exception of the applications in biology, physics, and so forth, Volterra integral equations often appear in some mathematical economic problems, for example, the relationships between capital and investment which include memory effects (in [1], the present stock of capital depends on the history of investment strategies over a period of time). And the simplest way to describe such memory effects is through Volterra integral operators. Based on the importance of Volterra integral equations, we will study a stochastic optimal control problem about a class of nonlinear stochastic equations-forward-backward stochastic Volterra integral equations (FBSVIEs, for short). First we review the backgrounds of these two kinds of Volterra integral equations: forward stochastic Volterra integral equations (FSVIEs, for short) and backward stochastic Volterra integral equations (BSVIEs, for short).

Let $B(\cdot)$ be a standard $d$-dimensional Brownian motion defined on a complete filtered probability space $(\Omega, \mathscr{F}, \mathbb{F}, P)$, where $\mathbb{F}=\left\{\mathscr{F}_{t}\right\}_{t \geq 0}$ is its natural filtration generated by $B(\cdot)$ and augmented by all the $P$-null sets in $\mathscr{F}$. Consider the following FSVIE:

$$
\begin{aligned}
X(t)= & \varphi(t)+\int_{0}^{t} b(t, s, X(s)) d s \\
& +\int_{0}^{t} \sigma(t, s, X(s)) d B_{s}, \quad t \in[0, T] .
\end{aligned}
$$

The readers may refer to [2-13] and the references cited therein for the general results on FSVIEs. When studying the stochastic optimal control problems of FSVIEs, we need one kind of adjoint equations in order to derive a stochastic maximum principle. This new adjoint equation is actually a linear BSVIE. This motivates the investigation of the theory and applications of BSVIEs.

The following BSVIE was firstly introduced by Yong [14]:

$$
\begin{aligned}
Y(t)= & \psi(t)+\int_{t}^{T} g(t, s, Y(s), Z(s, t)) d s \\
& -\int_{t}^{T} Z(t, s) d B_{s}, \quad t \in[0, T],
\end{aligned}
$$

where $g: \Delta^{c} \times \mathbb{R}^{m} \times \mathbb{R}^{m \times d} \times \Omega \rightarrow \mathbb{R}^{m}$ and $\psi:[0, T] \times \Omega \rightarrow$ $\mathbb{R}^{m}$ are given maps with $\Delta^{c}=\left\{(t, s) \in[0, T]^{2} \mid t<s\right\}$. For each $t \in[0, T], \psi(t)$ is $\mathscr{F}_{T}$-measurable (Lin [15] studied (2) when $\psi(\cdot) \equiv \xi)$. It is obvious that BSVIE is a natural generalization of backward stochastic differential equation (BSDE, for short). Comparing with BSDEs, BSVIE still has its own features as listed in Yong $[14,16]$. One of the advantages is to study time-inconsistent phenomenon. As shown in Laibson [17] and Strotz [18], in the real world, time-inconsistent preference usually exists. At this point, one needs BSVIEs to generalize the so-called stochastic differential utility in [19] and dynamic risk measures (see [20-23]). Other applications 
are in the nonexponential discounting problems (see Ekeland and Lazrak [24] and Ekeland and Pirvu [25]) and timeinconsistent optimal control problem (see Yong [26, 27]). In $[26,27]$, Yong solved a time-inconsistent optimal control problem by introducing a family of $\mathrm{N}$-person noncooperative differential games and got an equilibrium control which was represented via a forward ordinary differential equation with a backward Riccati-Volterra integral equation.

As stated in Yong [28], $\psi(t)$ in BSVIE (2) could represent the total (nominal) wealth of certain portfolio which might be a combination of certain contingent claims (e.g., European style, which is mature at time $T$, is usually only $\mathscr{F}_{T^{-}}$ measurable) and some current cash flows, positions of stocks, mutual funds, bonds, and so on, at time $t$. So, in general, the position process $\psi(\cdot)$ is not necessarily $\mathbb{F}$-adapted, but a stochastic process is merely $\mathscr{F}_{T}$-measurable. And Yong gave an example to make this point more clear in [28]. Focusing on this kind of position process $\psi(\cdot)$, a class of convex/coherent dynamic risk measures was introduced by Yong in [28] to measure the risk dynamically. Hence, one kind of control problems appears: how to minimize the risk or how to maximize the utility. Wang and Shi [29] obtained a maximum principle for FBSVIEs without state constraints. In this paper, we study one kind of optimal control problems in which the state equations are governed by the following FBSVIEs:

$$
\begin{aligned}
X(t)= & f(t)+\int_{0}^{t} b(t, s, X(s), u(s)) d s \\
& +\int_{0}^{t} \sigma(t, s, X(s), u(s)) d B_{s}, \quad t \in[0, T], \\
Y(t)= & \psi(t)+\int_{t}^{T} g(t, s, X(s), Y(s), Z(s, t), u(s)) d s \\
& -\int_{t}^{T} Z(t, s) d B_{s} .
\end{aligned}
$$

By choosing admissible controls $(u, \psi)$, we will maximize the following objective functional:

$$
\begin{aligned}
J(\psi, u):=E\left[\int_{0}^{T} \int_{t}^{T} l_{2}(t, s, X(s), Y(s),\right. \\
Z(s, t), u(s)) d s d t \\
+\int_{0}^{T} \int_{0}^{t} l_{1}(t, s, X(s), u(s)) d s d t \\
+\int_{0}^{T} q(\psi(t)) d t+h(X(T)) \\
\left.+\int_{0}^{T} k(Y(s)) d s\right] .
\end{aligned}
$$

Our formulation has the following new features.

(i) A strong assumption that $g(t, \cdot, \cdot, \cdot, \cdot, \cdot)$ in (3) is $\mathscr{F}_{t^{-}}$ measurable is given in [29]. By applying the duality principle introduced in Yong [28], we overcome this restriction and assume a natural condition that $g(\cdot, s, \cdot, \cdot, \cdot, \cdot)$ is $\mathscr{F}_{s}$-measurable.

(ii) $\psi$ in (3) is the terminal state of the BSVIE. In our formulation $\psi$ is also regarded as a control and our control is a pair $(u, \psi)$. In mathematical finance, such kind of controls often appears as "consumptioninvestment plan" (see [30]). For the recent progress of studying this kind of control, we refer the reader to [31-34]. We also impose constraints on the state process $Y(\cdot)$ and $\psi$.

(iii) We consider the double integral in the cost functional (4) in theory. Some further studies on the applications are still under consideration.

In order to solve this optimal control problem, we adopt the terminal perturbation method, which was introduced in [31-33, 35-41]. Recently, the dual approach is applied to utility optimization problem with volatility ambiguity (see $[42,43])$. The basic idea is to perturb the terminal state $\psi$ and $u$ directly. By applying Ekeland's variational principle to tackle the state constraints, we derive a stochastic maximum principle which characterizes the optimal control. It is worthy to point out that in place of Itô's formula, we need two duality principles established by Yong in $[16,28]$ to obtain the above results.

This paper is organized as follows. First, we recall some elements of the theory of BSVIEs in Section 2. In Section 3, we formulate the stochastic optimization problem and prove a stochastic maximum principle. In Section 4, we give two examples. The first example is associated with the model we studied. The last example is about the "terminal" control $\psi(\cdot)$.

\section{Preliminaries}

Let $B(\cdot)$ be a $d$-dimensional Brownian motion defined on a complete filtered probability space $(\Omega, \mathscr{F}, \mathbb{F}, P)$, where $\mathbb{F}=$ $\left\{\mathscr{F}_{t}\right\}_{t \geq 0}$ is natural filtration generated by $B(\cdot)$ and augmented by all the $P$-null sets in $\mathscr{F}$; that is,

$$
\mathscr{F}_{t}=\sigma\left\{B_{r}, r \leq t\right\} \vee \mathcal{N}_{P}, \quad t \in[0, T]
$$

where $\mathcal{N}_{P}$ is the set of all $P$-null sets.

2.1. Notations. Here we keep on the definitions and notations for the spaces introduced in Yong [16].

For any $0 \leq R<S \leq T$, we denote

$$
\begin{aligned}
\Delta[R, S] & =\left\{(t, s) \in[R, S]^{2} \mid R \leq s \leq t \leq S\right\}, \\
\Delta^{c}[R, S] & =\left\{(t, s) \in[R, S]^{2} \mid R \leq t<s \leq S\right\} \\
& \equiv[R, S]^{2} \backslash \Delta[R, S] .
\end{aligned}
$$


For any $A, B \in \mathbb{R}^{m \times d}$, define the inner product $\langle A, B\rangle:=$ $\operatorname{tr}\left[A B^{T}\right]$ and

$$
\begin{aligned}
|A|^{2} & =\sum_{j=1}^{d}\left|a_{j}\right|^{2} \\
& =\sum_{i=1}^{m} \sum_{j=1}^{d} a_{i j}^{2}, \quad \forall A \equiv\left(a_{1}, \ldots, a_{d}\right) \equiv\left(a_{i j}\right) \in \mathbb{R}^{m \times d} .
\end{aligned}
$$

Let $S \in[0, T]$; define the following spaces:

(i) $L_{\mathscr{F}_{S}}^{p}(0, T):=\left\{\varphi:[0, T] \times \Omega \rightarrow \mathbb{R}^{m} \mid \varphi(\cdot)\right.$ is $\mathscr{B}([0, T]) \otimes \mathscr{F}_{S}$-measurable and $\left.E \int_{0}^{T}|\varphi(t)|^{p} d t<\infty\right\} ;$

(ii) $L_{\mathbb{F}}^{p}(0, T):=\left\{\varphi:[0, T] \times \Omega \rightarrow \mathbb{R}^{m} \mid \varphi(\cdot)\right.$ is $\mathbb{F}$-adapted and $\left.E \int_{0}^{T}|\varphi(t)|^{p} d t<\infty\right\}$;

(iii) $L^{p}\left(0, T ; L_{\mathbb{F}}^{2}(0, T)\right):=\left\{Z \quad: \quad[0, T]^{2} \times \Omega \rightarrow\right.$ $\mathbb{R}^{m \times d} \mid$ for almost all $t \in[0, T], Z(t, \cdot) \quad \in$ $\left.L_{\mathbb{F}}^{2}(0, T), \int_{0}^{T} E\left(\int_{0}^{T}|Z(t, s)|^{2} d s\right)^{p / 2} d t<\infty\right\} ;$

(iv) $L_{\mathbb{F}}^{\infty}\left(0, T ; \mathbb{R}^{n}\right):=\left\{\varphi \quad:[0, T] \times \Omega \rightarrow \mathbb{R}^{n} \mid\right.$ $\left.\operatorname{essup}_{\omega \in \Omega} \sup _{s \in[0, T]} \varphi(s, \omega)<\infty\right\} ;$

(v) $L^{\infty}\left([0, T] ; L_{\mathbb{F}}^{\infty}\left(0, T ; \mathbb{R}^{n \times n}\right)\right)$

$$
\begin{aligned}
& \left\{Z(t, \cdot) \quad \in \quad L_{\mathbb{F}}^{\infty}\left(0, T ; \mathbb{R}^{n \times n}\right)\right. \\
& \left.\operatorname{essup}_{\omega \in \Omega} \sup _{t \in[0, T]} \sup _{s \in[0, T]} Z(t, s, \omega)<\infty\right\} ;
\end{aligned}
$$

(vi) $\mathscr{H}^{p}[S, T]:=L_{\mathbb{F}}^{p}(S, T) \times L^{p}\left(S, T ; L_{\mathbb{F}}^{2}(S, T)\right)$.

2.2. Backward Stochastic Volterra Integral Equations. For the reader's convenience, we present some results of BSVIEs which we will use later.

Consider the following integral equation:

$$
\begin{aligned}
Y(t)= & \psi(t)+\int_{t}^{T} g(t, s, Y(s), Z(s, t)) d s \\
& -\int_{t}^{T} Z(t, s) d B_{s}, \quad t \in[0, T],
\end{aligned}
$$

where $\psi(\cdot) \in L_{\mathscr{F}_{T}}^{2}(0, T)$.

We assume the following.

(H) Let $g: \Omega \times \Delta^{c}[0, T] \times \mathbb{R}^{m} \times \mathbb{R}^{m \times d} \rightarrow \mathbb{R}^{m}$ be $\mathscr{F}_{T} \otimes \mathscr{B}\left(\Delta^{c} \times \mathbb{R}^{m} \times \mathbb{R}^{m \times d}\right)$-measurable such that $s \mapsto g(t, s, y, \zeta)$ is $\mathbb{F}$-progressively measurable for all $(t, y, \zeta) \in[0, T] \times \mathbb{R}^{m} \times \mathbb{R}^{m \times d}$ and

$$
E \int_{0}^{T}\left(\int_{t}^{T}|g(t, s, 0,0)| d s\right)^{2} d t<\infty .
$$

Moreover, $\forall(t, s) \in \Delta^{c}[0, T],(y, \zeta)$ and $(\bar{y}, \bar{\zeta}) \in$ $\mathbb{R}^{m} \times \mathbb{R}^{m \times d}$

$$
\begin{aligned}
& |g(t, s, y, \zeta)-g(t, s, \bar{y}, \bar{\zeta})| \\
& \quad \leq L(t, s)(|y-\bar{y}|+|\zeta-\bar{\zeta}|), \quad \text { a.s., }
\end{aligned}
$$

where $L: \Delta^{c}[0, T] \rightarrow \mathbb{R}$ is a deterministic function such that

$$
\sup _{t \in[0, T]} \int_{t}^{T} L(t, s)^{2+\varepsilon} d s<\infty, \quad \text { for some } \varepsilon>0 .
$$

The following $M$-solution of BSVIEs was introduced by Yong [16].

Definition 1. Let $S \in[0, T)$. A pair $(Y(\cdot), Z(\cdot, \cdot)) \in \mathscr{H}^{2}[S, T]$ is called an adapted $M$-solution of BSVIE (8) on $[S, T]$ if (8) holds in the usual Itô sense for almost all $t \in[S, T]$ and, in addition, the following equation holds:

$$
Y(t)=E\left[Y(t) \mid \mathscr{F}_{\mathcal{\delta}}\right]+\int_{S}^{t} Z(t, s) d B_{s}, \quad \text { a.e., } t \in[S, T] .
$$

For the proof of the following well-posedness results, the readers are referred to Yong [16].

Lemma 2. Let $(\mathbf{H})$ hold. Then for any $\psi(\cdot) \in L_{\mathscr{F}_{T}}^{2}(0, T)$, BSVIE (8) admits a unique adapted $M$-solution $(Y(\cdot), Z(\cdot, \cdot)) \in$ $\mathscr{H}^{2}[0, T]$ on $[0, T]$. Moreover the following estimate holds: $\forall S \in[0, T]$,

$$
\begin{aligned}
& \|Y(\cdot), Z(\cdot, \cdot)\|_{\mathscr{H}^{2}[S, T]}^{2} \\
& \quad \equiv E\left\{\int_{S}^{T}|Y(t)|^{2} d t+\iint_{S}^{T}|Z(t, s)|^{2} d s d t\right\} \\
& \quad \leq C E\left\{\int_{S}^{T}|\psi(t)|^{2} d t+E \int_{S}^{T}\left(\int_{t}^{T}\left|g_{0}(t, s)\right| d s\right)^{2} d t\right\} .
\end{aligned}
$$

Let $\bar{g}: \Omega \times[0, T] \times[0, T] \times \mathbb{R}^{m} \times \mathbb{R}^{m \times d} \rightarrow \mathbb{R}^{m}$ also satisfy (H) and $\psi(\cdot) \in L_{\mathscr{F}_{T}}^{2}(0, T)$ and $(\bar{Y}(\cdot), \bar{Z}(\cdot, \cdot)) \in \mathscr{H}^{2}[0, T]$ is the adapted $M$-solution of $(8)$ with $g$ and $\psi(\cdot)$ replaced by $\bar{g}$ and $\bar{\psi}(\cdot)$, respectively: then for all $S \in[0, T]$,

$$
\begin{aligned}
& E\left\{\int_{S}^{T}|Y(t)-\bar{Y}(t)|^{2} d t\right. \\
& \left.\quad+\iint_{S}^{T}|Z(t, s)-\bar{Z}(t, s)|^{2} d s d t\right\} \\
& \quad \leq C E\left\{\int_{S}^{T}|\psi(t)-\bar{\psi}(t)|^{2} d t\right. \\
& +\int_{S}^{T}\left(\int_{t}^{T} \mid g(t, s, Y(s), Z(s, t))\right. \\
& \left.\quad-\bar{g}(t, s, Y(s), Z(s, t)) \mid d s)^{2} d t\right\} .
\end{aligned}
$$

Yong proved the following two duality principles for linear SVIE and linear BSVIE in [16, 28], respectively. And they play a key role in deriving the maximum principle. 
Lemma 3. Let $A_{i}(\cdot, \cdot) \in L^{\infty}\left([0, T] ; L_{\mathbb{F}}^{\infty}\left(0, T ; \mathbb{R}^{d \times d}\right)\right)(i=$ $0,1, \ldots, d), \varphi(\cdot) \in L_{\mathbb{F}}^{2}\left(0, T ; \mathbb{R}^{d}\right)$, and $\psi(t) \in L^{2}\left((0, T) \times \Omega ; \mathbb{R}^{d}\right)$. Let $\xi(\cdot) \in L_{\mathbb{F}}^{2}\left(0, T ; \mathbb{R}^{d}\right)$ be the solution of the following FSVIE:

$$
\begin{aligned}
\xi(t)= & \varphi(t)+\int_{0}^{T} A_{0}(t, s) \xi(s) d s \\
& +\int_{0}^{t} \sum_{i=1}^{d} A_{i}(t, s) \xi(s) d B_{i}(s), \quad t \in[0, T]
\end{aligned}
$$

Let $(Y(\cdot), Z(\cdot, \cdot)) \in \mathscr{H}^{2}[0, T]$ be the adapted M-solution to the following BSVIE:

$$
\begin{aligned}
Y(t)= & \psi(t) \\
& +\int_{t}^{T}\left[A_{0}(s, t)^{T} Y(s)+\sum_{i=1}^{d} A_{i}(s, t)^{T} Z_{i}(s, t)\right] d s \\
& -\int_{t}^{T} Z(t, s) d B_{s}, \quad t \in[0, T] .
\end{aligned}
$$

Then the following relation holds:

$$
E \int_{0}^{T}\langle\xi(t), \psi(t)\rangle d t=E \int_{0}^{T}\langle\varphi(t), Y(t)\rangle d t .
$$

Lemma 4. Let $A_{i}(\cdot, \cdot) \in L^{\infty}\left([0, T] ; L_{\mathbb{F}}^{\infty}\left(0, T ; \mathbb{R}^{d \times d}\right)\right)(i=$ $0,1, \ldots, d), \varphi(\cdot) \in L_{\mathbb{F}}^{2}\left(0, T ; \mathbb{R}^{d}\right)$, and $\psi(t) \in L^{2}\left((0, T) \times \Omega ; \mathbb{R}^{d}\right)$. Suppose that $(Y(\cdot), Z(\cdot, \cdot)) \in \mathscr{H}^{2}(0, T)$ is the solution of the following linear BSVIE:

$$
\begin{aligned}
Y(t)= & \psi(t) \\
& +\int_{t}^{T}\left[A_{0}(t, s)^{T} Y(s)+\sum_{i=1}^{d} A_{i}(t, s)^{T} Z_{i}(s, t)\right] d s \\
& -\int_{t}^{T} Z(t, s) d B_{s}, \quad t \in[0, T],
\end{aligned}
$$

and suppose that $X(\cdot)$ is the solution of the following FSVIE:

$$
\begin{aligned}
X(t)= & \varphi(t)+\int_{0}^{T} A_{0}(s, t) X(s) d s \\
& +\int_{0}^{t} X(s) \sum_{i=1}^{d} E\left[A_{i}(s, t) \mid \mathscr{F}_{s}\right] d B_{i}(s), \quad t \in[0, T] .
\end{aligned}
$$

Then the following relation holds:

$$
E \int_{0}^{T}\langle X(t), \psi(t)\rangle d t=E \int_{0}^{T}\langle\varphi(t), Y(t)\rangle d t
$$

For the proofs of Lemmas 3 and 4 , the readers are referred to Theorem 5.1 in [16] and Theorem 3.1 in [28], respectively.

\section{Stochastic Optimization Problem}

3.1. One Kind of Stochastic Optimization Problems. Let $K, \bar{K}$ be nonempty convex subsets of $\mathbb{R}^{m}$; set

$$
\begin{gathered}
U[0, T]=\left\{u:[0, T] \times \Omega \longrightarrow \mathbb{R}^{m} \mid u(\cdot) \in L_{\mathbb{F}}^{2}(0, T),\right. \\
u(s) \in K, s \in[0, T], \text { a.e., a.s. }\}, \\
\mathcal{U}=\left\{(\psi(\cdot), u(\cdot)) \mid \psi(\cdot) \in L_{\mathscr{F}_{T}}^{2}(0, T), \psi(t) \in \bar{K},\right. \\
t \in[0, T], \text { a.e., a.s., } u(\cdot) \in U[0, T]\} .
\end{gathered}
$$

For any given control pair $(\psi(\cdot), u(\cdot)) \in \mathcal{U}$, we consider the following controlled integral equation:

$$
\begin{aligned}
X(t)= & f(t)+\int_{0}^{t} b(t, s, X(s), u(s)) d s \\
& +\int_{0}^{t} \sigma(t, s, X(s), u(s)) d B_{s}, \quad t \in[0, T], \\
Y(t)= & \psi(t)+\int_{t}^{T} g(t, s, X(s), Y(s), Z(s, t), u(s)) d s \\
& -\int_{t}^{T} Z(t, s) d B_{s},
\end{aligned}
$$

where $f(\cdot) \in L_{\mathbb{F}}^{2}(0, T)$ and $b: \Omega \times \Delta[0, T] \times \mathbb{R}^{m} \times K \rightarrow \mathbb{R}^{m}$, $\sigma: \Omega \times \Delta[0, T] \times \mathbb{R}^{m} \times K \rightarrow \mathbb{R}^{m \times d}$, and $g: \Omega \times \Delta^{c}[0, T] \times$ $\mathbb{R}^{m} \times \mathbb{R}^{m} \times \mathbb{R}^{m \times d} \times K \rightarrow \mathbb{R}^{m}$.

For each $(\psi(\cdot), u(\cdot)) \in \mathcal{U}$, define the following objective functional:

$$
\begin{aligned}
J(\psi(\cdot), u(\cdot)):=E[ & \int_{0}^{T} \int_{t}^{T} l_{2}(t, s, X(s), Y(s), \\
& \left.+\int_{0}^{T} \int_{0}^{t} l_{1}(t, t), u(s)\right) d s d t \\
& \left.+\int_{0}^{T} q(\psi(t)) d t+h(x)\right) d s d t \\
& \left.+\int_{0}^{T} k(Y(t)) d t\right],
\end{aligned}
$$

where $l_{1}: \Delta[0, T] \times \mathbb{R}^{m} \times K \rightarrow \mathbb{R}, l_{2}: \Delta^{c}[0, T] \times \mathbb{R}^{m} \times$ $\mathbb{R}^{m} \times \mathbb{R}^{m \times d} \times K \rightarrow \mathbb{R}$, and $q: \mathbb{R}^{m} \rightarrow \mathbb{R}, h: \mathbb{R}^{m} \rightarrow \mathbb{R}$, $k: \mathbb{R}^{m} \rightarrow \mathbb{R}$.

We assume the following.

$\left(\mathbf{A}_{1}\right) b, \sigma, g, l_{1}, l_{2}, q, h, k$ are continuous in their argument and continuously differentiable in the variables $(x, y, \zeta, u)$;

$\left(\mathbf{A}_{2}\right)$ the derivatives of $b, \sigma, g, h$ in $(x, y, \zeta, u)$ are bounded;

$\left(\mathbf{A}_{3}\right)$ the derivatives of $l_{1}, l_{2}$ in $(x, y, \zeta, u)$ are bounded by $C(1+|x|+|y|+|\zeta|+|u|)$, and the derivatives of $q$, $h, k$ in $x$ are bounded by $C(1+|x|)$; 
$\left(\mathbf{A}_{4}\right) g(t, s, x, y, \zeta, u)$ is $\mathscr{F}_{T} \otimes \mathscr{B}\left(\Delta^{c}[0, T] \times \mathbb{R}^{m} \times \mathbb{R}^{m} \times\right.$ $\left.\mathbb{R}^{m \times d} \times K\right)$-measurable such that $s \mapsto g(t, s, x, y, \zeta, u)$, $s \mapsto g_{i}(t, s, x, y, \zeta, u)$ are $\mathbb{F}$-progressively measurable for all $(t, x, y, \zeta, u) \in[0, T] \times \mathbb{R}^{m} \times \mathbb{R}^{m} \times \mathbb{R}^{m \times d} \times K, i=$ $x, y, \zeta, u$, and $E \int_{0}^{T}\left(\int_{t}^{T}|g(t, s, 0,0,0,0)| d s\right)^{2} d t<\infty$.

Under the assumptions $\left(\mathbf{A}_{1}\right),\left(\mathbf{A}_{2}\right)$, and $\left(\mathbf{A}_{4}\right)$, for any given $u(\cdot) \in U[0, T]$, the FSVIE in (22) has a unique solution $X^{u}(\cdot) \in L_{\mathbb{F}}^{2}(0, T)$. For any given $\psi(\cdot) \in L_{\mathscr{F}_{T}}^{2}(0, T)$, the BSVIE has a unique M-solution $\left(Y^{\psi, u}(\cdot), Z^{\psi, u}(\cdot, \cdot)\right) \in \mathscr{H}^{2}[0, T]$ associated with $(\psi(\cdot), u(\cdot))$. Hence, there exists a unique triple $\left(X^{u}(\cdot), Y^{\psi, u}(\cdot), Z^{\psi, u}(\cdot, \cdot)\right)$ satisfying $(22)$.

Now we formulate the optimization problem:

$$
\begin{array}{ll}
\text { Maximize } & J(\psi(\cdot), u(\cdot)) \\
\text { subject to } & (\psi(\cdot), u(\cdot)) \in \mathcal{U}, \\
& \int_{0}^{T} E Y^{\psi, u}(s) d s=a, \\
& E Y^{\psi, u}(t)=\rho(t), \quad \text { a.e }
\end{array}
$$

where $\rho:[0, T] \rightarrow \mathbb{R}^{m}$ is continuous and satisfies $\int_{0}^{T} \rho(t) d t=a, \int_{0}^{T}|\rho(t)|^{2} d t<\infty$.

3.2. Variational Equation. For $\left(\psi^{1}(\cdot), u^{1}(\cdot)\right),\left(\psi^{2}(\cdot), u^{2}(\cdot)\right) \in$ $\mathcal{U}$, we define a metric in $\mathcal{U}$ by

$$
\begin{gathered}
d\left(\left(\psi^{1}(\cdot), u^{1}(\cdot)\right),\left(\psi^{2}(\cdot), u^{2}(\cdot)\right)\right) \\
:=\left(E \int_{0}^{T}\left|\psi^{1}(s)-\psi^{2}(s)\right|^{2} d s\right. \\
\left.\quad+E \int_{0}^{T}\left|u^{1}(s)-u^{2}(s)\right|^{2} d s\right)^{1 / 2} .
\end{gathered}
$$

It is obvious that $(\mathcal{U}, d(\cdot, \cdot))$ is a complete metric space.

Let $\left(\psi^{*}(\cdot), u^{*}(\cdot)\right)$ be an optimal control pair to problem (24) and let $\left(X^{*}(\cdot), Y^{*}(\cdot), Z^{*}(\cdot, \cdot)\right)$ be the corresponding state processes of $(22)$. For any $(\psi(\cdot), u(\cdot)) \in \mathcal{U}, 0 \leq p \leq 1$, using the convexity of $\mathcal{U}$, we have

$$
\begin{aligned}
& \left(\psi^{p}(\cdot), u^{p}(\cdot)\right) \\
& \quad:=\left((1-p) \psi^{*}(\cdot)+p \psi(\cdot),(1-p) u^{*}(\cdot)+p u(\cdot)\right) \\
& \quad=\left(\psi^{*}(\cdot)+p\left(\psi(\cdot)-\psi^{*}(\cdot)\right), u^{*}(\cdot)+p\left(u(\cdot)-u^{*}(\cdot)\right)\right) \\
& \quad \in \mathcal{U} .
\end{aligned}
$$

We denote $\left(X^{p}(\cdot), Y^{p}(\cdot), Z^{p}(\cdot, \cdot)\right)$ by the solution of the corresponding FBSVIE $(22)$ with $(\psi(\cdot), u(\cdot))=\left(\psi^{p}(\cdot), u^{p}(\cdot)\right)$.
Consider the following FBSVIE:

$$
\begin{aligned}
\delta X(t)= & \int_{0}^{t} b_{u}^{*}(t, s) \widehat{u}(s) d s+\int_{0}^{t} \sigma_{u}^{*}(t, s) \widehat{u}(s) d B_{s} \\
& +\int_{0}^{t} b_{x}^{*}(t, s) \delta X(s) d s \\
& +\int_{0}^{t} \sigma_{x}^{*}(t, s) \delta X(s) d B_{s}, \quad t \in[0, T], \\
\delta Y(t)= & \widehat{\psi}(t) \\
& +\int_{t}^{T}\left[g_{x}^{*}(t, s) \delta X(s)+g_{y}^{*}(t, s) \delta Y(s)\right. \\
& -\int_{t}^{T} \delta Z(t, s) d B_{s},
\end{aligned}
$$

where $\widehat{\psi}(s)=\psi(s)-\psi^{*}(s), \widehat{u}(s)=u(s)-u^{*}(s), f_{k}^{*}(t, s)=$ $f_{k}\left(t, s, X^{*}(s), Y^{*}(s), Z^{*}(s, t), u^{*}(s)\right)$, and $k=x, y, \zeta, u, f=$ $b, \sigma, g$, respectively. This equation is called the variational equation.

From Lemma 2 and $\left(\mathbf{A}_{1}\right),\left(\mathbf{A}_{2}\right),\left(\mathbf{A}_{4}\right)$, it is easy to check that the variational equation $(27)$ has a unique solution $(\delta X(\cdot), \delta Y(\cdot), \delta Z(\cdot, \cdot)) \in L_{\mathbb{F}}^{2}(0, T) \times \mathscr{H}^{2}[0, T]$.

Now we define

$$
\begin{gathered}
\widetilde{X}^{p}(t)=p^{-1}\left[X^{p}(t)-X^{*}(t)\right]-\delta X(t), \\
\widetilde{Y}^{p}(t)=p^{-1}\left[Y^{p}(t)-Y^{*}(t)\right]-\delta Y(t), \\
\widetilde{Z}^{p}(t, s)=p^{-1}\left[Z^{p}(t, s)-Z^{*}(t, s)\right]-\delta Z(t, s) .
\end{gathered}
$$

To simplify the proof, we use the following notations:

$$
\begin{aligned}
& f^{p}(t, s)=f\left(t, s, X^{p}(s), Y^{p}(s), Z^{p}(s, t), u^{p}(s)\right), \\
& f^{*}(t, s)=f\left(t, s, X^{*}(s), Y^{*}(s), Z^{*}(s, t), u^{*}(s)\right),
\end{aligned}
$$

where $f=b, \sigma, g$, respectively. Similar to the arguments in $[29,32]$, we have the following lemma.

Lemma 5. Assume that $\left(\mathbf{A}_{1}\right),\left(\mathbf{A}_{2}\right)$, and $\left(\mathbf{A}_{4}\right)$ hold. One has

$$
\begin{gathered}
\lim _{p \rightarrow 0} E \int_{0}^{T}\left|\widetilde{X}^{p}(t)\right|^{2} d t=0, \\
\lim _{p \rightarrow 0} E \int_{0}^{T}\left|\widetilde{Y}^{p}(t)\right|^{2} d t=0, \\
\lim _{p \rightarrow 0} E \iint_{0}^{T}\left|\widetilde{Z}^{p}(t, s)\right|^{2} d s d t=0 .
\end{gathered}
$$


Proof. (1) We prove the first equality. By the FSVIEs in (22) and (27), we have

$$
\begin{aligned}
\widetilde{X}^{p}(t)= & \int_{0}^{t} \frac{1}{p}\left[b^{p}(t, s)-b^{*}(t, s)-p b_{x}^{*}(t, s) \delta X(s)\right. \\
& \left.\quad-p b_{u}^{*}(t, s) \widehat{u}(s)\right] d s \\
& +\int_{0}^{t} \frac{1}{p}\left[\sigma^{p}(t, s)-\sigma^{*}(t, s)-p \sigma_{x}^{*}(t, s) \delta X(s)\right. \\
= & \int_{0}^{t}\left[A_{1}^{p}(t, s) \widetilde{X}^{p}(s)+D_{1}^{p}(t, s)\right] d s \\
& +\int_{0}^{t}\left[A_{2}^{p}(t, s) \widetilde{X}^{p}(s)+D_{2}^{p}(t, s)\right] d B_{s},
\end{aligned}
$$

where

$$
\begin{gathered}
A_{1}^{p}(t, s):=\int_{0}^{1} b_{x}(t, s, L(p, \lambda, s), M(p, \lambda, s)) d \lambda, \\
A_{2}^{p}(t, s):=\int_{0}^{1} \sigma_{x}(t, s, L(p, \lambda, s), M(p, \lambda, s)) d \lambda, \\
B_{1}^{p}(t, s):=\int_{0}^{1} b_{u}(t, s, L(p, \lambda, s), M(p, \lambda, s)) d \lambda, \\
B_{2}^{p}(t, s):=\int_{0}^{1} \sigma_{u}(t, s, L(p, \lambda, s), M(p, \lambda, s)) d \lambda, \\
D_{1}^{p}(t, s):=\left[A_{1}^{p}(t, s)-b_{x}^{*}(t, s)\right] \delta X(s) \\
\quad+\left[B_{1}^{p}(t, s)-b_{u}^{*}(t, s)\right] \widehat{u}(s), \\
D_{2}^{p}(t, s):=\left[A_{2}^{p}(t, s)-\sigma_{x}^{*}(t, s)\right] \delta X(s) \\
\quad+\left[B_{2}^{p}(t, s)-\sigma_{u}^{*}(t, s)\right] \widehat{u}(s), \\
L(p, \lambda, s):=X^{*}(s)+\lambda\left(X^{p}(s)-X^{*}(s)\right), \\
M(p, \lambda, s):=u^{*}(s)+\lambda\left(u^{p}(s)-u^{*}(s)\right) .
\end{gathered}
$$

Therefore, we have

$$
\begin{aligned}
E \int_{0}^{T} e^{-r t}\left|\widetilde{X}^{p}(t)\right|^{2} d t & \\
\leq & C E \int_{0}^{T} e^{-r t} \int_{0}^{T}\left(\left|A_{1}^{p}(t, s)\right|^{2}+\left|A_{2}^{p}(t, s)\right|^{2}\right)\left|\widetilde{X}^{p}(s)\right|^{2} d s d t \\
& +C E \int_{0}^{T} e^{-r t} \int_{0}^{T}\left(\left|D_{1}^{p}(t, s)\right|^{2}+\left|D_{2}^{p}(t, s)\right|^{2}\right) d s d t \\
\leq & \frac{C}{r} E \int_{0}^{T} e^{-r t}\left|\widetilde{X}^{p}(t)\right|^{2} d t \\
& +C E \iint_{0}^{T} e^{-r t}\left(\left|D_{1}^{p}(t, s)\right|^{2}+\left|D_{2}^{p}(t, s)\right|^{2}\right) d s d t .
\end{aligned}
$$

By choosing a proper $r$ such that $C / r<1$, we have

$$
\begin{aligned}
& E \int_{0}^{T}\left|\widetilde{X}^{p}(t)\right|^{2} d t \\
& \quad \leq C E \iint_{0}^{T} e^{r(T-t)}\left(\left|D_{1}^{p}(t, s)\right|^{2}+\left|D_{2}^{p}(t, s)\right|^{2}\right) d s d t .
\end{aligned}
$$

Applying Lebesgue's dominated convergence theorem, we have

$$
\lim _{p \rightarrow 0} E \iint_{0}^{T}\left|D_{i}^{p}(t, s)\right|^{2} d s d t=0, \quad i=1,2 .
$$

So,

$$
\lim _{p \rightarrow 0} E \int_{0}^{T}\left|\widetilde{X}^{p}(t)\right|^{2} d t=0
$$

(2) By the BSVIEs in (22) and (27), we have

$$
\begin{aligned}
\widetilde{Y}^{p}(t)=\int_{t}^{T} \frac{1}{p}\left[g^{p}(t, s)-g^{*}(t, s)-p g_{x}^{*}(t, s) \delta X(s)\right. \\
\quad-p g_{y}^{*}(t, s) \delta Y(s)-p g_{\zeta}^{*}(t, s) \delta Z(s, t) \\
\left.\quad-p g_{u}^{*}(t, s) \widehat{u}(s)\right] d s \\
-\int_{t}^{T} \widetilde{Z}^{p}(t, s) d B_{s}, \quad t \in[0, T] .
\end{aligned}
$$

Let

$$
\begin{gathered}
N(p, \lambda, s):=Y^{*}(s)+\lambda\left(Y^{p}(s)-Y^{*}(s)\right), \\
P(p, \lambda, t, s):=Z^{*}(s, t)+\lambda\left(Z^{p}(s, t)-Z^{*}(s, t)\right), \\
C_{1}^{p}(t, s):=\int_{0}^{1} g_{x}(t, s, L(p, \lambda, s), N(p, \lambda, s), \\
P(p, \lambda, t, s), M(p, \lambda, s)) d \lambda, \\
C_{2}^{p}(t, s):=\int_{0}^{1} g_{y}(t, s, L(p, \lambda, s), N(p, \lambda, s), \\
C_{3}^{p}(t, s):=\int_{0}^{1} g_{\zeta}(t, s, L(p, \lambda, s), N(p, \lambda, s), \\
P(p, \lambda, t, s), M(p, \lambda, s)) d \lambda, M(p, \lambda, s)) d \lambda, \\
C_{4}^{p}(t, s):=\int_{0}^{1} g_{u}(t, s, L(p, \lambda, s), N(p, \lambda, s), \\
+\left[C_{3}^{p}(t, s)-g_{\zeta}^{*}(t, s)\right] \delta Z(s, t) \\
\left.\left.+\left[C_{4}^{p}(t, s)-g_{u}^{*}(t, s)\right] \widehat{u}(s) . \lambda, t, s\right), M(p, \lambda, s)\right) d \lambda, \\
D^{p}(t, s):=\left[\begin{array}{l}
\left.C_{1}^{p}(t, s)-g_{x}^{*}(t, s)\right] \delta X(s) \\
+
\end{array}\right.
\end{gathered}
$$


Thus,

$$
\begin{aligned}
\widetilde{Y}^{p}(t)=\int_{t}^{T}\left[C_{1}^{p}(t, s) \widetilde{X}^{p}(s)+C_{2}^{p}(t, s) \widetilde{Y}^{p}(s)\right. \\
\left.+C_{3}^{p}(t, s) \widetilde{Z}^{p}(s, t)+D^{p}(t, s)\right] d s \\
-\int_{t}^{T} \widetilde{Z}^{p}(t, s) d B_{s}, \quad t \in[0, T] .
\end{aligned}
$$

In Lemma 2, we take $\psi=0, g_{0}(t, s)=C_{1}^{p}(t, s) \widetilde{X}^{p}(s)+D^{p}(t, s)$. Then

$$
\begin{gathered}
\left\|\left(\widetilde{Y}^{p}(t), \widetilde{Z}^{p}(t, s)\right)\right\|_{\mathscr{H}^{2}[0, T]}^{2} \\
=E\left[\int_{0}^{T}\left|\widetilde{Y}^{p}(t)\right|^{2} d s+\iint_{0}^{T}\left|\widetilde{Z}^{p}(t, s)\right|^{2} d s d t\right] \\
\leq C E \int_{0}^{T}\left[\left(\int_{t}^{T}\left|C_{1}^{p}(t, s) \widetilde{X}^{p}(s)\right| d s\right)^{2}\right. \\
\left.+\left(\int_{t}^{T}\left|D^{p}(t, s)\right| d s\right)^{2}\right] d t .
\end{gathered}
$$

Applying Lebesgue's dominated convergence theorem, we have

$$
\lim _{p \rightarrow 0} E \int_{0}^{T}\left(\int_{t}^{T}\left|D^{p}(t, s)\right| d s\right)^{2} d t \longrightarrow 0
$$

Using the obtained first result, we can get the desired results.

3.3. Variational Inequality. In this subsection, using Ekeland's variational principle (see [44]), we get the variational inequality.

Lemma 6 (Ekeland's variational principle). Let $(V, d(\cdot, \cdot))$ be a complete metric space and let $F(\cdot): V \rightarrow R$ be a proper lower semicontinuous function bounded from below. Suppose that, for some $\varepsilon>0$, there exists $u \in V$ satisfying $F(u) \leq$ $\inf _{v \in V} F(v)+\varepsilon$. Then there exists $u_{\varepsilon} \in V$ such that

(i) $F\left(u_{\varepsilon}\right) \leq F(u)$,

(ii) $d\left(u, u_{\varepsilon}\right) \leq \varepsilon$,

(iii) $F(v)+\sqrt{\varepsilon} d\left(v, u_{\varepsilon}\right) \geq F\left(u_{\varepsilon}\right)$, for all $v \in V$.
Given the optimal control pair $\left(\psi^{*}(\cdot), u^{*}(\cdot)\right) \in \mathcal{U}$, introduce a mapping $F_{\varepsilon}(\cdot): \mathscr{U} \rightarrow \mathbb{R}$ by

$$
\begin{aligned}
F_{\varepsilon}(\psi(\cdot), u(\cdot)) & \left\{\left|\int_{0}^{T} E Y(t) d t-a\right|^{2}+\int_{0}^{T}|E Y(t)-\rho(t)|^{2} d t\right. \\
& +\left\{\operatorname { m a x } \left(0, \int_{0}^{T} E k\left(Y^{*}(s)\right) d s\right.\right. \\
& +\left\{\max \left(0, E h\left(X^{*}(T)\right)-E h(X(T))+\varepsilon\right)\right\}^{2} \\
& +\left\{\operatorname { m a x } \left(0, \int_{0}^{T} E q\left(\psi^{*}(t)\right) d t\right.\right. \\
& \left.\left.+\int_{0}^{T} E q(\psi(t)) d t+\varepsilon\right)\right\}^{2} \\
& +\left\{\operatorname { m a x } \left(0, \int_{0}^{T} \int_{0}^{t} E l_{1}^{*}(t, s) d s d t\right.\right. \\
& \left.\left.-\int_{0}^{T} \int_{t}^{T} \int_{0}^{t} E l_{1}(t, s) d s d t+\varepsilon\right)\right\}^{2} \\
& +\int_{t}^{T} E l_{2}^{*}(t, s) d s d t
\end{aligned}
$$

where $l_{i}^{*}(t, s)=l_{i}\left(t, s, X^{*}(s), Y^{*}(s), Z^{*}(s, t), u^{*}(s)\right), l_{i}(t, s)=$ $l_{i}(t, s, X(s), Y(s), Z(s, t), u(s)), i=1,2, \varepsilon$, is an arbitrary positive constant and $l_{i}, q, h, k$ satisfy $\left(\mathbf{A}_{1}\right),\left(\mathbf{A}_{2}\right)$, and $\left(\mathbf{A}_{3}\right)$.

Remark 7. Under $\left(\mathbf{A}_{1}\right)-\left(\mathbf{A}_{4}\right)$, from the well-posedness of BSVIEs (Lemma 2) as well as the proof of Lemma 5, we know that $F_{\varepsilon}(\cdot, \cdot)$ is a continuous function on $\mathcal{U}$.

Theorem 8. Let $\left(\psi^{*}(\cdot), u^{*}(\cdot)\right) \in \mathcal{U}$ be the optimal control pair. Under the assumptions $\left(\mathbf{A}_{1}\right)-\left(\mathbf{A}_{4}\right)$, there exists a deterministic function $h_{0}(\cdot) \in \mathbb{R}^{m}, \bar{h}_{0} \in \mathbb{R}^{m}, \bar{h}_{1}, h_{1}, h_{2}, h_{3}, h_{4} \in \mathbb{R}, h_{1}, h_{2}$, $h_{3}, h_{4} \leq 0,\left|\bar{h}_{0}\right|+\left|h_{0}(\cdot)\right|+\left|\bar{h}_{1}\right|+\left|h_{1}\right|+\left|h_{2}\right|+\left|h_{3}\right|+\left|h_{4}\right| \neq 0$ such that the following variational inequality holds:

$$
\begin{aligned}
\int_{0}^{T} E & \left\langle h_{0}(t)+\bar{h}_{0}, \delta Y(t)\right\rangle d t \\
& +\bar{h}_{1} \int_{0}^{T} E\left\langle q_{x}\left(\psi^{*}(t)\right), \widehat{\psi}(t)\right\rangle d t \\
& +h_{1} E\left\langle h_{x}\left(X^{*}(T)\right), \delta X(T)\right\rangle \\
& +h_{2} \int_{0}^{T} E\left\langle k_{y}\left(Y^{*}(s)\right), \delta Y(s)\right\rangle d s
\end{aligned}
$$




$$
\begin{aligned}
& +h_{3} \int_{0}^{T} \int_{0}^{t} E\left\langle l_{1 x}^{*}(t, s), \delta X(s)\right\rangle d s d t \\
& +h_{3} \int_{0}^{T} \int_{0}^{t} E\left\langle l_{1 u}^{*}(t, s)^{T}, \widehat{u}^{*}(s)\right\rangle d s d t \\
& +h_{4} \int_{0}^{T} \int_{t}^{T} E\left\langle l_{2 x}^{*}(t, s), \delta X(s)\right\rangle d s d t \\
& +h_{4} \int_{0}^{T} \int_{t}^{T} E\left\langle l_{2 y}^{*}(t, s), \delta Y(s)\right\rangle d s d t \\
& +h_{4} \int_{0}^{T} \int_{t}^{T} E\left\langle l_{2 \zeta}^{*}(t, s), \delta Z(s, t)\right\rangle d s d t \\
& +h_{4} \int_{0}^{T} \int_{t}^{T} E\left\langle l_{2 u}^{*}(t, s), \widehat{u}^{*}(s)\right\rangle d s d t \geq 0,
\end{aligned}
$$

where $l_{i k}^{*}(t, s), i=1,2, k=x, y, \zeta, u$, is the derivative of $l_{i}^{*}(t, s)$ with respect to $k$, respectively.

Proof. It is easy to check that the following properties hold:

(i) $F_{\varepsilon}\left(\psi^{*}(\cdot), u^{*}(\cdot)\right)=\sqrt{5} \varepsilon$,

(ii) $F_{\varepsilon}(\psi(\cdot), u(\cdot))>0$, for all $(\psi(\cdot), u(\cdot)) \in \mathcal{U}$,

(iii) $F_{\varepsilon}\left(\psi^{*}(\cdot), u^{*}(\cdot)\right) \leq \inf _{(\psi, u) \in \mathcal{U}} F_{\varepsilon}(\psi(\cdot), u(\cdot))+\sqrt{5} \varepsilon$.

Then, from Lemma 6 (Ekeland's variational principle), we can find $\left(\psi^{\varepsilon}(\cdot), \mathcal{u}^{\varepsilon}(\cdot)\right) \in \mathcal{U}$, such that one has the following:

(i) $F_{\varepsilon}\left(\psi^{\varepsilon}(\cdot), u^{\varepsilon}(\cdot)\right) \leq F_{\varepsilon}\left(\psi^{*}(\cdot), u^{*}(\cdot)\right)$,

(ii) $d\left(\left(\psi^{\varepsilon}(\cdot), u^{\varepsilon}(\cdot)\right),\left(\psi^{*}(\cdot), u^{*}(\cdot)\right)\right) \leq \sqrt{5} \varepsilon$,

(iii) $F_{\varepsilon}(\psi(\cdot), u(\cdot))+\sqrt{\sqrt{5}} \varepsilon d\left((\psi(\cdot), u(\cdot)),\left(\psi^{\varepsilon}(\cdot), u^{\varepsilon}(\cdot)\right)\right) \geq$ $F_{\varepsilon}\left(\psi^{\varepsilon}(\cdot), u^{\varepsilon}(\cdot)\right)$, for all $(\psi(\cdot), u(\cdot)) \in \mathcal{U}$.

For each $(\psi(\cdot), u(\cdot)) \in \mathcal{U}$, we define

$$
\begin{aligned}
(\widehat{\psi}(\cdot), \widehat{u}(\cdot)) & :=\left(\psi(\cdot)-\psi^{*}(\cdot), u(\cdot)-u^{*}(\cdot)\right),\left(\widehat{\psi}^{\varepsilon}(\cdot), \widehat{u}^{\varepsilon}(\cdot)\right) \\
& :=\left(\psi(\cdot)-\psi^{\varepsilon}(\cdot), u(\cdot)-u^{\varepsilon}(\cdot)\right) ;
\end{aligned}
$$

then $\left(\psi_{p}^{\varepsilon}(\cdot), u_{p}^{\varepsilon}(\cdot)\right):=\left(\psi^{\varepsilon}(\cdot)+p \widehat{\psi}^{\varepsilon}(\cdot), u^{\varepsilon}(\cdot)+p \widehat{u}^{\varepsilon}(\cdot)\right) \in U$. Indeed, $\left(\psi^{\varepsilon}(\cdot), u^{\varepsilon}(\cdot)\right) \in \mathcal{U},\left(\widehat{\psi}^{\varepsilon}(\cdot)+\psi^{\varepsilon}(\cdot), \widehat{u}^{\varepsilon}(\cdot)+u^{\varepsilon}(\cdot)\right)=$ $(\psi(\cdot), u(\cdot)) \in \mathcal{U}$; then

$$
\begin{aligned}
\left(\psi_{p}^{\varepsilon}, u_{p}^{\varepsilon}\right):= & \left(\psi^{\varepsilon}(\cdot)+p \widehat{\psi}^{\varepsilon}(\cdot), u^{\varepsilon}(\cdot)+p \widehat{u}^{\varepsilon}(\cdot)\right) \\
= & \left((1-p) \psi^{\varepsilon}(\cdot)+p\left(\widehat{\psi}^{\varepsilon}(\cdot)+\psi^{\varepsilon}(\cdot)\right),(1-p) u^{\varepsilon}(\cdot)\right. \\
& \left.+p\left(\widehat{u}^{\varepsilon}(\cdot)+u^{\varepsilon}(\cdot)\right)\right) \in \mathcal{U} .
\end{aligned}
$$

Let $\left(X_{p}^{\varepsilon}(\cdot), Y_{p}^{\varepsilon}(\cdot), Z_{p}^{\varepsilon}(\cdot, \cdot)\right)$ (resp., $\left.\left(X^{\varepsilon}(\cdot), Y^{\varepsilon}(\cdot), Z^{\varepsilon}(\cdot, \cdot)\right)\right)$ be the solution of BSVIE (22) with $(\psi(\cdot), u(\cdot))=\left(\psi_{p}^{\varepsilon}(\cdot), u_{p}^{\varepsilon}(\cdot)\right)$ (resp., $\left.(\psi(\cdot), u(\cdot))=\left(\psi^{\varepsilon}(\cdot), u^{\varepsilon}(\cdot)\right)\right)$. From Ekeland's variational principle, it follows that

$$
\begin{gathered}
F_{\varepsilon}\left(\psi_{p}^{\varepsilon}(\cdot), u_{p}^{\varepsilon}(\cdot)\right)+\sqrt{\sqrt{5} \varepsilon d}\left(\left(\psi_{p}^{\varepsilon}(\cdot), u_{p}^{\varepsilon}(\cdot)\right),\left(\psi^{\varepsilon}(\cdot), u^{\varepsilon}(\cdot)\right)\right) \\
-F_{\varepsilon}\left(\psi^{\varepsilon}(\cdot), u^{\varepsilon}(\cdot)\right) \geq 0 .
\end{gathered}
$$

We consider the following variational equation:

$$
\begin{aligned}
\delta X^{\varepsilon}(t)= & \int_{0}^{t} b_{u}^{\varepsilon}(t, s) \widehat{u}^{\varepsilon}(s) d s+\int_{0}^{t} \sigma_{u}^{\varepsilon}(t, s) \widehat{u}^{\varepsilon}(s) d B_{s} \\
& +\int_{0}^{t} b_{x}^{\varepsilon}(t, s) \delta X^{\varepsilon}(s) d s+\int_{0}^{t} \sigma_{x}^{\varepsilon}(t, s) \delta X^{\varepsilon}(s) d B_{s}, \\
t \in[0, T], & \\
\delta Y^{\varepsilon}(t)=\widehat{\psi}^{\varepsilon}(t)+\int_{t}^{T}\left[g_{x}^{\varepsilon}(t, s) \delta X^{\varepsilon}(s)+g_{y}^{\varepsilon}(t, s) \delta Y^{\varepsilon}(s)\right. & +g_{\zeta}^{\varepsilon}(t, s) \delta Z^{\varepsilon}(s, t) \\
& \left.+g_{u}^{\varepsilon}(t, s)\left(u(s)-u^{\varepsilon}(s)\right)\right] d s \\
& -\int_{t}^{T} \delta Z^{\varepsilon}(t, s) d B_{s},
\end{aligned}
$$

where $f_{k}^{\varepsilon}(t, s)=f_{k}\left(t, s, X^{\varepsilon}(s), Y^{\varepsilon}(s), Z^{\varepsilon}(s, t), u^{\varepsilon}(s)\right), k=x, y$, $\zeta, u, f=b, \sigma, g$, respectively.

Similarly to Lemma 5, we have

$$
\begin{aligned}
& \operatorname{Lim}_{p \rightarrow 0} E \int_{0}^{T}\left|\frac{X_{p}^{\varepsilon}(t)-X^{\varepsilon}(t)}{p}-\delta X^{\varepsilon}(t)\right|^{2} d t=0, \\
& \lim _{p \rightarrow 0} E \int_{0}^{T}\left|\frac{Y_{p}^{\varepsilon}(t)-Y^{\varepsilon}(t)}{p}-\delta Y^{\varepsilon}(t)\right|^{2} d t=0,
\end{aligned}
$$

which leads to the following expansions:

$$
\begin{gathered}
E X_{p}^{\varepsilon}(t)-E X^{\varepsilon}(t)=p E \delta X^{\varepsilon}(t)+o(p), \\
E Y_{p}^{\varepsilon}(t)-E Y^{\varepsilon}(t)=p E \delta Y^{\varepsilon}(t)+o(p), \\
\int_{0}^{T}\left|E Y_{p}^{\varepsilon}(t)-\rho(t)\right|^{2} d t-\int_{0}^{T}\left|E Y^{\mathcal{\varepsilon}}(t)-\rho(t)\right|^{2} d t \\
=\int_{0}^{T} 2 p\left\langle E Y^{\varepsilon}(t)-\rho(t), E \delta Y^{\varepsilon}(t)\right\rangle d t+o(p) .
\end{gathered}
$$


Abstract and Applied Analysis

9

From $\left(\mathbf{A}_{1}\right)$, we have

$$
\begin{aligned}
& \int_{0}^{T} E q\left(\psi_{p}^{\varepsilon}(t)\right) d t-\int_{0}^{T} E q\left(\psi^{\varepsilon}(t)\right) d t \\
& =p \int_{0}^{T} E\left\langle q_{x}\left(\psi^{\varepsilon}(t)\right), \widehat{\psi}^{\varepsilon}(t)\right\rangle d t+o(p), \\
& E h\left(X_{p}^{\varepsilon}(T)\right)-E h\left(X^{\varepsilon}(T)\right) \\
& =p E\left\langle h_{x}\left(X^{\varepsilon}(T)\right), \delta X^{\varepsilon}(T)\right\rangle+o(p), \\
& \int_{0}^{T} E k\left(Y_{p}^{\varepsilon}(t)\right) d t-\int_{0}^{T} E k\left(Y^{\varepsilon}(t)\right) d t \\
& =p \int_{0}^{T}\left\langle E k_{y}\left(Y^{\varepsilon}(s)\right), \delta Y^{\varepsilon}(s)\right\rangle d s+o(p), \\
& \int_{0}^{T} \int_{0}^{t} E l_{1}^{p \varepsilon}(t, s) d s d t-\int_{0}^{T} \int_{0}^{t} E l_{1}^{\varepsilon}(t, s) d s d t \\
& =p \int_{0}^{T} \int_{0}^{t} E\left(\left\langle 1_{1 x}^{\varepsilon}(t, s), \delta X^{\varepsilon}(s)\right\rangle\right. \\
& \left.+\left\langle 1_{1 u}^{\varepsilon}(t, s), \widehat{u}^{\varepsilon}(s)\right\rangle\right) d s d t+o(p), \\
& \int_{0}^{T} \int_{t}^{T} E l_{2}^{p \varepsilon}(t, s) d s d t-\int_{0}^{T} \int_{0}^{t} E l_{2}^{\varepsilon}(t, s) d s d t \\
& =p \int_{0}^{T} \int_{t}^{T} E\left(\left\langle 1_{2 x}^{\varepsilon}(t, s), \delta X^{\varepsilon}(s)\right\rangle\right. \\
& +\left\langle 1_{2 y}^{\varepsilon}(t, s), \delta Y^{\varepsilon}(s)\right\rangle \\
& +\left\langle 1_{2 z}^{\varepsilon}(t, s), \delta Z^{\varepsilon}(s, t)\right\rangle \\
& \left.+\left\langle 1_{2 u}^{\varepsilon}(t, s), \widehat{u}^{\varepsilon}(s)\right\rangle\right) d s d t+o(p) .
\end{aligned}
$$

Furthermore, the following expansions hold:

$$
\begin{aligned}
& \left.\left|\int_{0}^{T} E Y_{p}^{\varepsilon}(t) d t-a\right|^{2}-\mid \int_{0}^{T} E Y^{\varepsilon}(t) d t-a\right)\left.\right|^{2} \\
& =2\left\langle\int_{0}^{T} E Y^{\varepsilon}(t) d t-a, \int_{0}^{T} E Y_{p}^{\varepsilon}(t) d t-\int_{0}^{T} E Y^{\varepsilon}(t) d t\right\rangle \\
& \quad+o(p) \\
& =2 p\left[\int_{0}^{T} E Y^{\varepsilon}(t) d t-a\right] \int_{0}^{T} E \delta Y^{\varepsilon}(t) d t+o(p), \\
& {\left[\int_{0}^{T} E q\left(\psi^{*}(t)\right) d t-\int_{0}^{T} E q\left(\psi_{p}^{\varepsilon}(t)\right) d t+\varepsilon\right]^{2}} \\
& \quad-\left[\int_{0}^{T} E q\left(\psi^{*}(t)\right) d t-\int_{0}^{T} E q\left(\psi^{\varepsilon}(t)\right) d t+\varepsilon\right]^{2} \\
& =2\left\langle\int_{0}^{T} q\left(\psi^{\varepsilon}(t)\right) d t\right. \\
& \quad-\int_{0}^{T} q\left(\psi_{p}^{\varepsilon}(t)\right) d t, \int_{0}^{T} E q\left(\psi^{*}(t)\right) d t
\end{aligned}
$$

$$
\begin{aligned}
& \left.-\int_{0}^{T} E q\left(\psi^{\varepsilon}(t)\right) d t+\varepsilon\right\rangle+o(p) \\
& =-2 p\left[\int_{0}^{T} E q\left(\psi^{*}(t)\right) d t-\int_{0}^{T} E q\left(\psi^{\varepsilon}(t)\right) d t+\varepsilon\right] \\
& \times \int_{0}^{T} E\left\langle q_{x}\left(\psi^{\varepsilon}(t)\right), \widehat{\psi}^{\varepsilon}(t)\right\rangle d t+o(p), \\
& {\left[E h\left(X^{*}(T)-E h\left(X_{p}^{\varepsilon}(T)\right)\right)+\varepsilon\right]^{2}} \\
& -\left[E h\left(X^{*}(T)-E h\left(X^{\varepsilon}(T)\right)\right)+\varepsilon\right]^{2} \\
& =2\left\langle E h\left(X^{\varepsilon}(T)\right)-E h\left(X_{p}^{\varepsilon}(T)\right), E h\left(X^{*}(T)\right)\right. \\
& \left.-E h\left(X^{\varepsilon}(T)\right)+\varepsilon\right\rangle+o(p) \\
& =-2 p\left[E h\left(X^{*}(T)\right)-E h\left(X^{\varepsilon}(T)\right)+\varepsilon\right] \\
& \times E\left\langle h_{x}\left(X^{\varepsilon}(T)\right), \delta X^{\varepsilon}(T)\right\rangle+o(p), \\
& {\left[\int_{0}^{T} E k\left(Y^{*}(s)\right) d s-\int_{0}^{T} E k\left(Y_{p}^{\varepsilon}(s)\right) d s+\varepsilon\right]^{2}} \\
& -\left[\int_{0}^{T} E k\left(Y^{*}(s)\right) d s-\int_{0}^{T} E k\left(Y^{\varepsilon}(s)\right) d s+\varepsilon\right]^{2} \\
& =-2\left\langle\int_{0}^{T} E k\left(Y^{\varepsilon}(s)\right) d s\right. \\
& -\int_{0}^{T} E k\left(Y_{p}^{\varepsilon}(s)\right) d s, \int_{0}^{T} E k\left(Y^{*}(s)\right) d s \\
& \left.-\int_{0}^{T} E k\left(Y^{\varepsilon}(s)\right) d s+\varepsilon\right\rangle+o(p) \\
& =-2 p\left[\int_{0}^{T} E k\left(Y^{*}(s)\right) d s-\int_{0}^{T} E k\left(Y^{\varepsilon}(s)\right) d s+\varepsilon\right] \\
& \times \int_{0}^{T} E\left\langle k_{y}\left(Y^{\varepsilon}(s)\right), \delta Y^{\varepsilon}(s)\right\rangle d s+o(p), \\
& \left(\int_{0}^{T} \int_{0}^{t} E l_{1}^{*}(t, s) d s d t-\int_{0}^{T} \int_{0}^{t} E l_{1}^{p \varepsilon}(t, s) d s d t+\varepsilon\right)^{2} \\
& -\left(\int_{0}^{T} \int_{0}^{t} E l_{1}^{*}(t, s) d s d t-\int_{0}^{T} \int_{0}^{t} E l_{1}^{\varepsilon}(t, s) d s d t+\varepsilon\right)^{2} \\
& =2\left\langle\int_{0}^{T} \int_{0}^{t} E l_{1}^{\varepsilon}(t, s) d s d t\right. \\
& -\int_{0}^{T} \int_{0}^{t} E l_{1}^{p \varepsilon}(t, s) d s d t, \int_{0}^{T} \int_{0}^{t} E l_{1}^{*}(t, s) d s d t \\
& \left.-\int_{0}^{T} \int_{0}^{t} E l_{1}^{\varepsilon}(t, s) d s d t+\varepsilon\right\rangle+o(p) \\
& =-2 p\left[\int_{0}^{T} \int_{0}^{t} E l_{1}^{*}(t, s) d s d t\right.
\end{aligned}
$$




$$
\begin{aligned}
& \left.-\int_{0}^{T} \int_{0}^{t} E l_{1}^{\varepsilon}(t, s) d s d t+\varepsilon\right] \\
& \times\left[\int _ { 0 } ^ { T } \int _ { 0 } ^ { t } E \left(\left\langle l_{1 x}^{\varepsilon}(t, s), \delta X^{\varepsilon}(s)\right\rangle\right.\right. \\
& \left.\left.+\left\langle l_{1 u}^{\varepsilon}(t, s), \widehat{u}^{\varepsilon}(s)\right\rangle\right) d s d t\right]+o(p) \\
& \left(\int_{0}^{T} \int_{t}^{T} E l_{2}^{*}(t, s) d s d t-\int_{0}^{T} \int_{t}^{T} E l_{2}^{p \varepsilon}(t, s) d s d t+\varepsilon\right)^{2} \\
& -\left(\int_{0}^{T} \int_{t}^{T} E l_{2}^{*}(t, s) d s d t-\int_{0}^{T} \int_{t}^{T} E l_{2}^{\varepsilon}(t, s) d s d t+\varepsilon\right)^{2} \\
& =2\left\langle\int_{0}^{T} \int_{t}^{T} E l_{2}^{\varepsilon}(t, s) d s d t\right. \\
& -\int_{0}^{T} \int_{t}^{T} E l_{2}^{p \varepsilon}(t, s) d s d t, \int_{0}^{T} \int_{t}^{T} E l_{2}^{*}(t, s) d s d t \\
& \left.-\int_{0}^{T} \int_{t}^{T} E l_{2}^{\varepsilon}(t, s) d s d t+\varepsilon\right\rangle+o(p) \\
& =-2 p\left[\int_{0}^{T} \int_{t}^{T} E l_{2}^{*}(t, s) d s d t-\int_{0}^{T} \int_{t}^{T} E l_{2}^{\varepsilon}(t, s) d s d t+\varepsilon\right] \\
& \cdot\left[\int _ { 0 } ^ { T } \int _ { t } ^ { T } E \left(\left\langle l_{2 x}^{\varepsilon}(t, s), \delta X^{\varepsilon}(s)\right\rangle+\left\langle l_{2 y}^{\varepsilon}(t, s), \delta Y^{\varepsilon}(s)\right\rangle\right.\right. \\
& +\left\langle l_{2 z}^{\varepsilon}(t, s), \delta Z^{\varepsilon}(s, t)\right\rangle \\
& \left.\left.+\left\langle l_{2 u}^{\varepsilon}(t, s), \widehat{u}^{\varepsilon}(s)\right\rangle\right) d s d t\right]+o(p)
\end{aligned}
$$

For the given $\varepsilon$, we consider the following cases.

Case 1. There exists $r>0$ such that, for any $p \in(0, r)$,

$$
\begin{gathered}
\int_{0}^{T} E q\left(\psi^{*}(t)\right) d t-\int_{0}^{T} E q\left(\psi_{p}^{\varepsilon}(t)\right) d t+\varepsilon>0, \\
E h\left(X^{*}(T)\right)-E h\left(X_{p}^{\varepsilon}(T)\right)+\varepsilon>0, \\
\int_{0}^{T} E k\left(Y^{*}(s)\right) d s-\int_{0}^{T} E k\left(Y_{p}^{\varepsilon}(s)\right) d s+\varepsilon>0, \\
\int_{0}^{T} \int_{0}^{t} E l_{1}^{*}(t, s) d s d t-\int_{0}^{T} \int_{0}^{t} E l_{1}^{p \varepsilon}(t, s) d s d t+\varepsilon>0, \\
\int_{0}^{T} \int_{t}^{T} E l_{2}^{*}(t, s) d s d t-\int_{0}^{T} \int_{t}^{T} E l_{2}^{p \varepsilon}(t, s) d s d t+\varepsilon>0 .
\end{gathered}
$$

Then

$$
\lim _{p \rightarrow 0} \frac{F_{\varepsilon}\left(\psi_{p}^{\varepsilon}(\cdot), u_{p}^{\varepsilon}(\cdot)\right)-F_{\varepsilon}\left(\psi^{\varepsilon}(\cdot), u^{\varepsilon}(\cdot)\right)}{p}
$$

$$
\begin{aligned}
=\lim _{p \rightarrow 0} \frac{1}{F_{\varepsilon}\left(\psi_{p}^{\varepsilon}(\cdot), u_{p}^{\varepsilon}(\cdot)\right)+F_{\varepsilon}\left(\psi^{\varepsilon}(\cdot), u^{\varepsilon}(\cdot)\right)} \\
. \frac{F_{\varepsilon}^{2}\left(\psi_{p}^{\varepsilon}(\cdot), u_{p}^{\varepsilon}(\cdot)\right)-F_{\varepsilon}^{2}\left(\psi^{\varepsilon}(\cdot), u^{\varepsilon}(\cdot)\right)}{p} \\
=\frac{1}{F_{\varepsilon}\left(\psi^{\varepsilon}(\cdot), u^{\varepsilon}(\cdot)\right)} \\
\times\left\{\int_{0}^{T} E Y^{\varepsilon}(t) d t-a, \int_{0}^{T} E \delta Y^{\varepsilon}(t) d t\right\rangle \\
\quad+\int_{0}^{T}\left\langle E Y^{\varepsilon}(t)-\rho(t), E \delta Y^{\varepsilon}(t)\right\rangle d t \\
\quad-\left[\int_{0}^{T} E q\left(\psi^{*}(t)\right) d t-\int_{0}^{T} E q\left(\psi^{\varepsilon}(t)\right) d t+\varepsilon\right] \\
\quad \times\left[\int_{0}^{T} E\left\langle q_{x}\left(\psi^{\varepsilon}(t)\right), \widehat{\psi}^{\varepsilon}(t)\right\rangle d t\right. \\
\quad-\left[E h\left(X^{*}(T)\right)-E h\left(X^{\varepsilon}(T)\right)+\varepsilon\right] \\
\times \\
\quad \times\left[\int_{0}^{T} E\left\langle k_{y}\left(Y^{\varepsilon}(s)\right), \delta Y^{\varepsilon}(s)\right\rangle d s\right. \\
\quad\left[\int_{0}^{T} E k\left(Y^{*}(s)\right), \delta X^{\varepsilon}(T)\right\rangle
\end{aligned}
$$$$
\times\left[\int _ { 0 } ^ { T } \int _ { 0 } ^ { t } E \left(\left\langle l_{1 x}^{\varepsilon}(t, s), \delta X^{\varepsilon}(s)\right\rangle\right.\right.
$$$$
\left.\left.+\left\langle l_{1 u}^{\varepsilon}(t, s), \widehat{u}^{\varepsilon}(s)\right\rangle\right) d s d t\right]
$$$$
-\left[\int_{0}^{T} \int_{t}^{T} E l_{2}^{*}(t, s) d s d t\right.
$$$$
\left.-\int_{0}^{T} \int_{t}^{T} E l_{2}^{\varepsilon}(t, s) d s d t+\varepsilon\right]
$$$$
\times\left[\int _ { 0 } ^ { T } \int _ { t } ^ { T } E \left(\left\langle l_{2 x}^{\varepsilon}(t, s), \delta X^{\varepsilon}(s)\right\rangle\right.\right.
$$$$
+\left\langle l_{2 y}^{\varepsilon}(t, s), \delta Y^{\varepsilon}(s)\right\rangle
$$$$
+\left\langle l_{2 z}^{\varepsilon}(t, s), \delta Z^{\varepsilon}(s, t)\right\rangle
$$$$
\left.\left.\left.+\left\langle l_{2 u}^{\varepsilon}(t, s), \widehat{u}^{\varepsilon}(s)\right\rangle\right) d s d t\right]\right\} .
$$ 
Set

$$
\begin{aligned}
\vec{h}_{\varepsilon}^{0}= & \frac{\int_{0}^{T} E Y^{\varepsilon}(t) d t-a}{F_{\varepsilon}\left(\psi^{\varepsilon}(\cdot), u^{\varepsilon}(\cdot)\right)}, \quad h_{\varepsilon}^{0}(t)=\frac{E Y^{\varepsilon}(t)-\rho(t)}{F_{\varepsilon}\left(\psi^{\varepsilon}(\cdot), u^{\varepsilon}(\cdot)\right)} \\
\bar{h}_{\varepsilon}^{1}= & -\frac{1}{F_{\varepsilon}\left(\psi^{\varepsilon}(\cdot), u^{\varepsilon}(\cdot)\right)} \\
& \times\left[\int_{0}^{T} E q\left(\psi^{*}(t)\right) d t-\int_{0}^{T} E q\left(\psi^{\varepsilon}(t)\right) d t+\varepsilon\right] \\
< & 0, \\
h_{\varepsilon}^{1}= & -\frac{1}{F_{\varepsilon}\left(\psi^{\varepsilon}(\cdot), u^{\varepsilon}(\cdot)\right)} \\
h_{\varepsilon}^{2}= & -\frac{1}{F_{\varepsilon}\left(\psi^{\varepsilon}(\cdot), u^{\varepsilon}(\cdot)\right)} \\
& \times\left[\int_{0}^{T} E k\left(Y^{*}(s)\right) d s-\int_{0}^{T} E k\left(Y^{\varepsilon}(s)\right) d s+\varepsilon\right] \\
< & 0, \\
h_{\varepsilon}^{3}=- & \frac{\times}{F_{\varepsilon}\left(\psi^{\varepsilon}(\cdot), u^{\varepsilon}(\cdot)\right)} \\
& \times\left[\int_{0}^{T} \int_{0}^{t} E l_{1}^{*}(t, s) d s d t-\int_{0}^{T} E l_{2}^{*}(t, s) d s d t-\int_{0}^{t} \int_{t}^{\varepsilon}(t, s) d s d t+\varepsilon\right] \\
< & 0, \\
h_{\varepsilon}^{4}=- & \left.\frac{1}{F_{\varepsilon}\left(\psi^{\varepsilon}(\cdot), u^{\varepsilon}(\cdot)\right)}(t, s) d s d t+\varepsilon\right] \\
& \\
& \\
&
\end{aligned}
$$

Then it follows from (46) that

$$
\begin{aligned}
& \int_{0}^{T} E\left\langle h_{\varepsilon}^{0}(t)+\bar{h}_{\varepsilon}^{0}, \delta Y^{\varepsilon}(t)\right\rangle d t \\
&+\bar{h}_{\varepsilon}^{1} \int_{0}^{T} E\left\langle q_{x}\left(\psi^{\varepsilon}(t)\right), \widehat{\psi}^{\varepsilon}(t)\right\rangle d t \\
&+h_{\varepsilon}^{1} E\left\langle h_{x}\left(X^{\varepsilon}(T)\right), \delta X^{\varepsilon}(T)\right\rangle \\
&+ h_{\varepsilon}^{2} \int_{0}^{T} E\left\langle k_{y}\left(Y^{\varepsilon}(s)\right), \delta Y^{\varepsilon}(s)\right\rangle d s \\
&+ h_{\varepsilon}^{3}\left[\int _ { 0 } ^ { T } \int _ { 0 } ^ { t } E \left(\left\langle l_{1 x}^{\varepsilon}(t, s), \delta X^{\varepsilon}(s)\right\rangle\right.\right. \\
&\left.\left.+\left\langle l_{1 u}^{\varepsilon}(t, s), \widehat{u}^{\varepsilon}(s)\right\rangle\right) d s d t\right]
\end{aligned}
$$

$$
\begin{gathered}
+h_{\varepsilon}^{4}\left[\int _ { 0 } ^ { T } \int _ { t } ^ { T } E \left(\left\langle l_{2 x}^{\varepsilon}(t, s), \delta X^{\varepsilon}(s)\right\rangle\right.\right. \\
+\left\langle l_{2 y}^{\varepsilon}(t, s), \delta Y^{\varepsilon}(s)\right\rangle \\
+\left\langle l_{2 z}^{\varepsilon}(t, s), \delta Z^{\varepsilon}(s, t)\right\rangle \\
\left.\left.+\left\langle l_{2 u}^{\varepsilon}(t, s), \widehat{u}^{\varepsilon}(s)\right\rangle\right) d s d t\right] \\
\geq-\sqrt{\sqrt{5} \varepsilon}\left[E \int_{0}^{T}\left|\widehat{\psi}^{\varepsilon}(t)\right|^{2} d t+E \int_{0}^{T}\left|\widehat{u}^{\varepsilon}(t)\right|^{2} d t\right]^{1 / 2}
\end{gathered}
$$

Case 2. There exists a positive sequence $\left\{p_{n}\right\}$, which satisfies $p_{n} \rightarrow 0$ such that

$$
\begin{gathered}
\int_{0}^{T} E q\left(\psi^{*}(t)\right) d t-\int_{0}^{T} E q\left(\psi_{p}^{\varepsilon}(t)\right) d t+\varepsilon \leq 0, \\
E h\left(X^{*}(T)\right)-E h\left(X_{p_{n}}^{\varepsilon}(T)\right)+\varepsilon \leq 0, \\
\int_{0}^{T} E k\left(Y^{*}(s)\right) d s-\int_{0}^{T} E k\left(Y_{p_{n}}^{\varepsilon}(s)\right) d s+\varepsilon \leq 0, \\
\int_{0}^{T} \int_{0}^{t} E l_{1}^{*}(t, s) d s d t-\int_{0}^{T} \int_{0}^{t} E l_{1}^{p_{n} \varepsilon}(t, s) d s d t+\varepsilon \leq 0, \\
\int_{0}^{T} \int_{t}^{T} E l_{2}^{*}(t, s) d s d t-\int_{0}^{T} \int_{t}^{T} E l_{2}^{p_{n} \varepsilon}(t, s) d s d t+\varepsilon \leq 0 .
\end{gathered}
$$

From the definition of $F_{\varepsilon}$, for enough large $n$,

$$
\begin{aligned}
F_{\varepsilon}\left(\psi_{p_{n}}^{\varepsilon}(\cdot), u_{p_{n}}^{\varepsilon}(\cdot)\right)= & \left\{\left|Y_{p_{n}}^{\varepsilon}(0)-\rho(0)\right|^{2}\right. \\
& \left.+\int_{0}^{T}\left|E Y_{p_{n}}^{\varepsilon}(t)-\rho(t)\right|^{2} d t\right\}^{1 / 2} .
\end{aligned}
$$

Since $F_{\varepsilon}(\cdot)$ is continuous, we know that $F_{\varepsilon}\left(\psi^{\varepsilon}(\cdot), u^{\varepsilon}(\cdot)\right)=$ $\left\{\left|\int_{0}^{T} E Y^{\varepsilon}(t) d t-a\right|^{2}+\int_{0}^{T}\left|E Y^{\varepsilon}(t)-\rho(t)\right|^{2} d t\right\}^{1 / 2}$.

$$
\begin{aligned}
\lim _{n \rightarrow \infty} & \frac{F_{\varepsilon}\left(\psi_{p_{n}}^{\varepsilon}(\cdot), u_{p_{n}}^{\varepsilon}(\cdot)\right)-F_{\varepsilon}\left(\psi^{\varepsilon}(\cdot), u^{\varepsilon}(\cdot)\right)}{p_{n}} \\
= & \lim _{n \rightarrow \infty} \frac{1}{F_{\varepsilon}\left(\psi_{p_{n}}^{\varepsilon}(\cdot), u_{p_{n}}^{\varepsilon}(\cdot)\right)+F_{\varepsilon}\left(\psi^{\varepsilon}(\cdot), u^{\varepsilon}(\cdot)\right)} \\
& \cdot \frac{F_{\varepsilon}^{2}\left(\psi_{p_{n}}^{\varepsilon}(\cdot), u_{p_{n}}^{\varepsilon}(\cdot)\right)-F_{\varepsilon}^{2}\left(\psi^{\varepsilon}(\cdot), u^{\varepsilon}(\cdot)\right)}{p_{n}} \\
= & \frac{1}{F_{\varepsilon}\left(\psi^{\varepsilon}(\cdot), u^{\varepsilon}(\cdot)\right)} \\
& \times\left\{\left\langle\int_{0}^{T} E Y^{\varepsilon}(t) d t-a, \int_{0}^{T} E \delta Y^{\varepsilon}(t) d t\right\rangle\right. \\
& \left.+\int_{0}^{T}\left\langle E Y^{\varepsilon}(t)-\rho(t), E \delta Y^{\varepsilon}(t)\right\rangle d t\right\} .
\end{aligned}
$$


Similar to Case 1, it follows from (46) that

$$
\begin{aligned}
& \int_{0}^{T} E\left\langle\vec{h}_{\varepsilon}^{0}+h_{\varepsilon}^{0}(t), \delta Y^{\varepsilon}(t)\right\rangle d t \\
& \quad \geq-\sqrt{\sqrt{5} \varepsilon}\left[E \int_{0}^{T}\left|\widehat{\psi}^{\varepsilon}(t)\right|^{2} d t+E \int_{0}^{T}\left|\widehat{u}^{\varepsilon}(t)\right|^{2} d t\right]^{1 / 2},
\end{aligned}
$$

where $\bar{h}_{\varepsilon}^{0}=\left(\int_{0}^{T} E Y^{\varepsilon}(t) d t-a\right) /\left(F_{\varepsilon}\left(\psi^{\varepsilon}(\cdot), u^{\varepsilon}(\cdot)\right)\right), h_{\varepsilon}^{0}(t)=$ $\left(E Y^{\varepsilon}(t)-\rho(t)\right) /\left(F_{\varepsilon}\left(\psi^{\varepsilon}(\cdot), u^{\varepsilon}(\cdot)\right)\right), \bar{h}_{\varepsilon}^{1}=h_{\varepsilon}^{1}=h_{\varepsilon}^{2}=h_{\varepsilon}^{3}=h_{\varepsilon}^{4}=0$. Similarly, we can prove that (55) still holds for the other thirty cases.

In summary, for given $\varepsilon$, we have the following:

(i) (55) holds,

(ii) $\bar{h}_{\varepsilon}^{1} \leq 0, h_{\varepsilon}^{1} \leq 0, h_{\varepsilon}^{2} \leq 0, h_{\varepsilon}^{4} \leq 0, h_{\varepsilon}^{5} \leq 0$,

(iii) $\left|h_{\varepsilon}^{0}\right|^{2}+\int_{0}^{T}\left|h_{\varepsilon}^{0}(t)\right|^{2} d t+\left|\bar{h}_{\varepsilon}^{1}\right|^{2}+\left|h_{\varepsilon}^{1}\right|^{2}+\left|h_{\varepsilon}^{2}\right|^{2}+\left|h_{\varepsilon}^{3}\right|^{2}+\left|h_{\varepsilon}^{4}\right|^{2}=1$.

Hence there is a subsequence $\left(\bar{h}_{\varepsilon_{n}}^{0}, h_{\varepsilon_{n}}^{0}(\cdot), \bar{h}_{\varepsilon_{n}}^{1}, h_{\varepsilon_{n}}^{1}, h_{\varepsilon_{n}}^{2}, h_{\varepsilon_{n}}^{3}, h_{\varepsilon_{n}}^{4}\right)$ of $\left(\bar{h}_{\varepsilon}^{0}, h_{\varepsilon}^{0}(\cdot), \bar{h}_{\varepsilon}^{1}, h_{\varepsilon}^{1}, h_{\varepsilon}^{2}, h_{\varepsilon}^{3}, h_{\varepsilon}^{4}\right)$, such that $\bar{h}_{\varepsilon_{n}}^{0} \rightarrow \bar{h}_{0}, h_{\varepsilon_{n}}^{0}(\cdot) \rightarrow$ $h_{0}(\cdot), \bar{h}_{\varepsilon_{n}}^{1} \rightarrow \bar{h}_{1}, h_{\varepsilon_{n}}^{1} \rightarrow h_{1}, h_{\varepsilon_{n}}^{2} \rightarrow h_{2}, h_{\varepsilon_{n}}^{3} \rightarrow h_{3}, h_{\varepsilon_{n}}^{4} \rightarrow h_{4}$. Since $\bar{h}_{\varepsilon}^{1}, h_{\varepsilon}^{1}, h_{\varepsilon}^{2}, h_{\varepsilon}^{3}, h_{\varepsilon}^{4} \leq 0$, we have $\bar{h}_{1}, h_{1}, h_{2}, h_{3}, h_{4} \leq 0$.

Because of $d\left(\left(\psi^{\varepsilon}(\cdot), u^{\varepsilon}(\cdot)\right),\left(\psi^{*}(\cdot), u^{*}(\cdot)\right)\right) \leq \sqrt{5} \varepsilon$, we have $\left(\psi^{\varepsilon}(\cdot), u^{\varepsilon}(\cdot)\right) \rightarrow\left(\psi^{*}(\cdot), u^{*}(\cdot)\right)$ in $\mathcal{U}$. Therefore, from the wellposedness of FBSVIEs, it is easy to check $\delta X^{\varepsilon}(\cdot) \rightarrow \delta X(\cdot)$, $\delta Y^{\varepsilon}(\cdot) \rightarrow \delta Y(\cdot)$, as $\varepsilon \rightarrow 0$. Furthermore, as $\varepsilon \rightarrow 0$,

$$
\begin{aligned}
\left|E\left\langle h_{x}\left(X^{\varepsilon}(T)\right), \delta X^{\varepsilon}(T)\right\rangle-E\left\langle h_{x}\left(X^{*}(T)\right), \delta X(T)\right\rangle\right| \\
=\mid E\left\langle h_{x}\left(X^{\varepsilon}(T)\right), \delta X^{\varepsilon}(T)-\delta X(T)\right\rangle \\
\quad+E\left\langle h_{x}\left(X^{\varepsilon}(T)\right)-h_{x}\left(X^{*}(T)\right), \delta X(T)\right\rangle \mid \longrightarrow 0 .
\end{aligned}
$$

Indeed, together with the Schwarz inequality, using the boundedness of $h_{x}$, we can get that the limit of the first part goes to 0 ; from the continuity $h_{x}$, we get that the second part also goes to 0 . Similarly, as $\varepsilon \rightarrow 0$, we have $\int_{0}^{T} E\left\langle q_{x}\left(\psi^{\varepsilon}(t)\right), \widehat{\psi}^{\varepsilon}(t)\right\rangle d t \rightarrow \int_{0}^{T} E\left\langle q_{x}\left(\psi^{*}(t)\right), \widehat{\psi}(t)\right\rangle d t$, $\left\langle k_{y}\left(Y^{\varepsilon}(0)\right), \delta Y^{\varepsilon}(0)\right\rangle \rightarrow\left\langle k_{y}\left(Y^{*}(0)\right), \delta Y(0)\right\rangle$, and

$$
\begin{aligned}
& \int_{0}^{T} \int_{0}^{t} E\left\langle l_{1 x}^{\varepsilon}(t, s), \delta X^{\varepsilon}(s)\right\rangle d s d t \\
& \quad \longrightarrow \int_{0}^{T} \int_{0}^{t} E\left\langle l_{1 x}^{*}(t, s), \delta X(s)\right\rangle d s d t
\end{aligned}
$$

$$
\begin{gathered}
\int_{0}^{T} \int_{0}^{t} E\left\langle l_{1 u}^{\varepsilon}(t, s), \widehat{u}^{\varepsilon}(s)\right\rangle d s d t \\
\longrightarrow \int_{0}^{T} \int_{0}^{t} E\left\langle l_{1 u}^{*}(t, s), \widehat{u}^{*}(s)\right\rangle d s d t, \\
\int_{0}^{T} \int_{t}^{T} E\left\langle l_{2 x}^{\varepsilon}(t, s), \delta X^{\varepsilon}(s)\right\rangle d s d t \\
\longrightarrow \int_{0}^{T} \int_{t}^{T} E\left\langle l_{2 x}^{*}(t, s), \delta X(s)\right\rangle d s d t, \\
\int_{0}^{T} \int_{t}^{T} E\left\langle l_{2 y}^{\varepsilon}(t, s), \delta Y^{\varepsilon}(s)\right\rangle d s d t \\
\longrightarrow \int_{0}^{T} \int_{t}^{T} E\left\langle l_{2 y}^{*}(t, s), \delta Y(s)\right\rangle d s d t, \\
\int_{0}^{T} \int_{t}^{T} E\left\langle l_{2 z}^{\varepsilon}(t, s), \delta Z^{\varepsilon}(s, t)\right\rangle d s d t \\
\longrightarrow \int_{0}^{T} \int_{t}^{T} E\left\langle l_{2 z}^{*}(t, s), \delta Z(s, t)\right\rangle d s d t, \\
\int_{0}^{T} \int_{t}^{T} E\left\langle l_{2 u}^{\varepsilon}(t, s), \widehat{u}^{\varepsilon}(s)\right\rangle d s d t \\
\quad \longrightarrow\left\langle l_{2 u}^{*}(t, s), \widehat{u}^{*}(s)\right\rangle d s d t .
\end{gathered}
$$

Letting $\varepsilon \rightarrow 0$ in (55), the result holds. The proof is completed.

3.4. Maximal Principle. We introduce the adjoint equation as follows:

$$
\begin{aligned}
m(t)= & A(t)+\int_{t}^{T}\left[b_{x}^{*}(s, t)^{T} m(s)+\sigma_{x}^{*}(s, t)^{T} n(s, t)\right] d s \\
& -\int_{t}^{T} n(t, s) d B_{s}, \\
p(t)= & B(t)+\int_{0}^{t} g_{y}^{*}(s, t)^{T} p(s) d s \\
& +\int_{0}^{t} E\left[g_{\zeta}^{*}(s, t)^{T} \mid \mathscr{F}_{s}\right] p(s) d B_{s}, \quad t \in[0, T],
\end{aligned}
$$

where

$$
\begin{gathered}
A(t)=h_{1} b_{x}^{*}(T, t)^{T} h_{x}\left(X^{*}(T)\right)+h_{1} \sigma_{x}^{*}(T, t)^{T} \pi(t) \\
+\int_{0}^{t} g_{x}^{*}(s, t)^{T} p(s) d s, \\
B(t)=h_{0}(t)+\bar{h}_{0}+h_{2} k_{y}\left(Y^{*}(t)\right), \\
\text { and } h_{x}\left(X^{*}(T)\right)=E h_{x}\left(X^{*}(T)\right)+\int_{0}^{T} \pi(s) d B_{s} .
\end{gathered}
$$

By the duality principles, we get the following theorem.

Theorem 9. Assume that $\left(\mathbf{A}_{1}\right)-\left(\mathbf{A}_{4}\right)$ hold and $l_{1}, l_{2}=$ 0 . Let $\left(\psi^{*}(\cdot), u^{*}(\cdot)\right)$ be the optimal control pair; let 
$\left(X^{*}(\cdot), Y^{*}(\cdot), Z^{*}(\cdot, \cdot)\right)$ be the corresponding optimal trajectory. Then there exists a deterministic function $h_{0}(\cdot) \in \mathbb{R}^{m}$, $\bar{h}_{0} \in \mathbb{R}^{m}, \bar{h}_{1}, h_{1}, h_{2} \leq 0$ such that $\forall(\psi(\cdot), u(\cdot)) \in \mathcal{U}$,

$$
\begin{aligned}
\langle p(t)+ & \left.\bar{h}_{1} q_{x}\left(\psi^{*}(t)\right), \psi(t)-\psi^{*}(t)\right\rangle \\
+ & \int_{t}^{T}\left\langle g_{u}^{*}(t, s)^{T} p(t), u(s)-u^{*}(s)\right\rangle d s \\
+ & h_{1}\left\langle b_{u}^{*}(T, t)^{T} E\left[h_{x}\left(X^{*}(T)\right)\right]\right. \\
& \left.+\sigma_{u}^{*}(T, t)^{T} \pi(t), u(t)-u^{*}(t)\right\rangle \\
+ & \int_{t}^{T}\left\langle b_{u}^{*}(s, t)^{T} m(s)\right. \\
& \left.+\sigma_{u}^{*}(s, t)^{T} n(s, t), u(t)-u^{*}(t)\right\rangle d s
\end{aligned}
$$

$\geq 0$, a.e., a.s,

where $(m(\cdot), n(\cdot, \cdot), p(\cdot))$ is the solution of the adjoint equation (62).

Proof. From the duality principles (Lemmas 3 and 4), we have the following relations:

$$
\begin{aligned}
& E \int_{0}^{T}\langle A(t), \delta X(t)\rangle d t \\
& =E \int_{0}^{T}\left\langle m(t), \int_{0}^{T} b_{u}^{*}(t, s) \widehat{u}(s) d s\right. \\
& \left.\quad+\int_{0}^{T} \sigma_{u}^{*}(t, s) \widehat{u}(s) d B_{s}\right\rangle d t \\
& =E \int_{0}^{T} \int_{t}^{T} m(s)^{T} b_{u}^{*}(s, t) \widehat{u}(t) d s d t \\
& \quad+E \int_{0}^{T} \int_{t}^{T} \sum_{i=1}^{d} n_{i}(s, t)^{T}\left(\sigma_{u}^{*}\right)^{i}(s, t) \widehat{u}(t) d s d t, \\
& E \int_{0}^{T}\langle B(t), \delta Y(t)\rangle d t \\
& =E \int_{0}^{T}\langle p(t), \widehat{\psi}(t) \\
& \left.\quad+\int_{t}^{T}\left[g_{x}^{*}(t, s) \delta X(s)+g_{u}^{*}(t, s) \widehat{u}(s)\right] d s\right\rangle d t .
\end{aligned}
$$

Combined with the variational inequality (Theorem 8 ), we get

$$
\begin{aligned}
0 \leq & \int_{0}^{T} E\left\langle\bar{h}_{0}+h_{0}(t), \delta Y(t)\right\rangle d t \\
& +\bar{h}_{1} \int_{0}^{T} E\left\langle q_{x}\left(\psi^{*}(t)\right), \widehat{\psi}(t)\right\rangle d t \\
& +h_{1} E\left\langle h_{x}\left(X^{*}(T)\right), \delta X(T)\right\rangle
\end{aligned}
$$

$$
\begin{aligned}
& +h_{2} \int_{0}^{T} E\left\langle k_{y}\left(Y^{*}(s)\right), \delta Y(s)\right\rangle d s \\
& =\int_{0}^{T} E\left\langle\bar{h}_{0}+h_{0}(t), \delta Y(t)\right\rangle d t \\
& +\bar{h}_{1} \int_{0}^{T} E\left\langle q_{x}\left(\psi^{*}(t)\right), \widehat{\psi}(t)\right\rangle d t \\
& +h_{1} E\left\langle h_{x}\left(X^{*}(T)\right), \delta X(T)\right\rangle \\
& +h_{2} \int_{0}^{T} E\left\langle k_{y}\left(Y^{*}(s)\right), \delta Y(s)\right\rangle d s \\
& +E \int_{0}^{T} \int_{t}^{T} m(s)^{T} b_{u}^{*}(s, t) \widehat{u}(t) d s d t \\
& +E \int_{0}^{T} \int_{t}^{T} \sum_{i=1}^{d} n_{i}(s, t)^{T}\left(\sigma_{u}^{*}\right)^{i}(s, t) \widehat{u}(t) d s d t \\
& -E \int_{0}^{T}\langle A(t), \delta X(t)\rangle d t \\
& +E \int_{0}^{T}\langle p(t), \widehat{\psi}(t) \\
& \left.+\int_{t}^{T}\left[g_{x}^{*}(t, s) \delta X(s)+g_{u}^{*}(t, s) \widehat{u}(s)\right] d s\right\rangle d t \\
& -E \int_{0}^{T}\langle B(t), \delta Y(t)\rangle d t \\
& =\int_{0}^{T} E\left\langle\bar{h}_{0}+h_{0}(t), \delta Y(t)\right\rangle d t \\
& +\bar{h}_{1} \int_{0}^{T} E\left\langle q_{x}\left(\psi^{*}(t)\right), \widehat{\psi}(t)\right\rangle d t \\
& +h_{1} E\left\langle h_{x}\left(X^{*}(T)\right), \int_{0}^{T} b_{u}^{*}(T, s) \widehat{u}(s) d s\right. \\
& \left.+\int_{0}^{T} \sigma_{u}^{*}(T, s) \widehat{u}(s) d B_{s}\right\rangle \\
& +h_{1} E\left\langle h_{x}\left(X^{*}(T)\right), \int_{0}^{T} b_{x}^{*}(T, s) \delta X(s) d s\right. \\
& \left.+\int_{0}^{T} \sigma_{x}^{*}(T, s) \delta X(s) d B_{s}\right\rangle \\
& +h_{2} \int_{0}^{T} E\left\langle k_{y}\left(Y^{*}(s)\right), \delta Y(s)\right\rangle d s \\
& +E \int_{0}^{T} \int_{t}^{T} m(s)^{T} b_{u}^{*}(s, t) \widehat{u}(t) d s d t \\
& +E \int_{0}^{T} \int_{t}^{T} \sum_{i=1}^{d} n_{i}(s, t)^{T}\left(\sigma_{u}^{*}\right)^{i}(s, t) \widehat{u}(t) d s d t \\
& -E \int_{0}^{T}\langle A(t), \delta X(t)\rangle d t
\end{aligned}
$$




$$
\begin{gathered}
+E \int_{0}^{T}\langle p(t), \widehat{\psi}(t) \\
\left.\quad+\int_{t}^{T}\left[g_{x}^{*}(t, s) \delta X(s)+g_{u}^{*}(t, s) \widehat{u}(s)\right] d s\right\rangle d t \\
+E \int_{0}^{T}\langle B(t), \delta Y(t)\rangle d t \\
=E \int_{0}^{T}\left\langle p(t)+\bar{h}_{1} q_{x}\left(\psi^{*}(t)\right), \widehat{\psi}(t)\right\rangle d t \\
+E \int_{0}^{T} \int_{t}^{T}\left\langle g_{u}^{*}(t, s)^{T} p(t), \widehat{u}(s)\right\rangle d s d t \\
+h_{1} E \int_{0}^{T}\left\langle b_{u}^{*}(T, t)^{T} E\left[h_{x}\left(X^{*}(T)\right)\right]\right. \\
\left.+\sum_{i=1}^{d}\left(\sigma_{u}^{*}\right)^{i}(T, t)^{T} \pi_{i}(t), \widehat{u}(t)\right\rangle d t \\
+E \int_{0}^{T} \int_{t}^{T}\left\langle b_{u}^{*}(s, t)^{T} m(s)\right. \\
\left.\quad+\sum_{i=1}^{d}\left(\sigma_{u}^{*}\right)^{i}(s, t)^{T} n_{i}(s, t), \widehat{u}(t)\right\rangle d s d t .
\end{gathered}
$$

Since the above holds for all $(\psi(\cdot), u(\cdot)) \in \mathcal{U}$, we obtain

$$
\begin{aligned}
\left\langle p(t)+\bar{h}_{1} q_{x}\left(\psi^{*}(t)\right), \psi(t)-\psi^{*}(t)\right\rangle & \\
+ & \int_{t}^{T}\left\langle g_{u}^{*}(t, s)^{T} p(t), u(s)-u^{*}(s)\right\rangle d s \\
+ & h_{1}\left\langle b_{u}^{*}(T, t)^{T} E\left[h_{x}\left(X^{*}(T)\right)\right]\right. \\
\quad & \left.\quad+\sigma_{u}^{*}(T, t)^{T} \pi(t), u(t)-u^{*}(t)\right\rangle \\
+\int_{t}^{T}\left\langle b_{u}^{*}(s, t)^{T} m(s)\right. & \left.\quad+\sigma_{u}^{*}(s, t)^{T} n(s, t), u(t)-u^{*}(t)\right\rangle d s \geq 0, \\
& \text { a.e., a.s. }
\end{aligned}
$$

When $l_{1}, l_{2} \neq 0$, the associated adjoint equation is

$$
\begin{aligned}
m(t)= & A(t)+\int_{t}^{T}\left[b_{x}^{*}(s, t)^{T} m(s)+\sigma_{x}^{*}(s, t)^{T} n(s, t)\right] d s \\
& -\int_{t}^{T} n(t, s) d B_{s}, \\
p(t)= & B(t)+\int_{0}^{T} g_{y}^{*}(s, t)^{T} p(s) d s
\end{aligned}
$$$$
+\int_{0}^{t} E\left[g_{\zeta}^{*}(s, t)^{T} \mid \mathscr{F}_{s}\right] p(s) d B_{s}, \quad t \in[0, T],
$$

where

$$
\begin{aligned}
A(t)= & h_{1} \sigma_{x}^{*}(T, t)^{T} \pi(t)+h_{1} b_{x}^{*}(T, t)^{T} h_{x}\left(X^{*}(T)\right) \\
& +\int_{0}^{T} g_{x}^{*}(s, t)^{T} p(s) d s \\
& +\int_{t}^{T} h_{3} l_{1 x}^{*}(s, t) d s+\int_{t}^{T} h_{4} l_{2 x}^{*}(s, t) d s, \\
B(t)= & h_{0}(t)+\bar{h}_{0}+h_{2} k_{y}\left(Y^{*}(t)\right)+\int_{0}^{T} h_{4} l_{2 y}^{*}(s, t) d s \\
& +\int_{0}^{t} h_{4} l_{2 \zeta}^{*}(s, t) d s,
\end{aligned}
$$

and $h_{x}\left(X^{*}(T)\right)=E h_{x}\left(X^{*}(T)\right)+\int_{0}^{T} \pi(s) d B_{s}$.

Similarly, we have the following maximum principle.

Theorem 10. Assume that $\left(\mathbf{A}_{1}\right)-\left(\mathbf{A}_{4}\right)$ hold. Let $\left(\psi^{*}(\cdot), u^{*}(\cdot)\right)$ be the optimal control pair; let $\left(X^{*}(\cdot), Y^{*}(\cdot), Z^{*}(\cdot, \cdot)\right)$ be the corresponding optimal trajectory. Then there exists a deterministic function $h_{0}(\cdot) \in \mathbb{R}^{m}, \bar{h}_{0} \in \mathbb{R}^{m}, \bar{h}_{1}, h_{1}, h_{2}, h_{3}, h_{4} \leq 0$ such that $\forall(\psi(\cdot), u(\cdot)) \in U$,

$$
\begin{aligned}
& \left\langle p(t)+\bar{h}_{1} q_{x}\left(\psi^{*}(t)\right), \psi(t)-\psi^{*}(t)\right\rangle \\
& +\int_{t}^{T}\left\langle g_{u}^{*}(t, s)^{T} p(t), u(s)-u^{*}(s)\right\rangle d s \\
& +h_{1}\left\langle b_{u}^{*}(T, t)^{T} E\left[h_{x}\left(X^{*}(T)\right)\right]\right. \\
& \left.\quad+\sigma_{u}^{*}(T, t)^{T} \pi(t), u(t)-u^{*}(t)\right\rangle \\
& +\int_{t}^{T}\left\langle b_{u}^{*}(s, t)^{T} m(s)+\sigma_{u}^{*}(s, t)^{T} n(s, t), u(t)-u^{*}(t)\right\rangle d s \\
& +h_{3} \int_{0}^{T}\left\langle l_{1 u}^{*}(t, s), u(s)-u^{*}(s)\right\rangle d s \\
& +h_{4} \int_{t}^{T}\left\langle l_{2 u}^{*}(t, s), u(s)-u^{*}(s)\right\rangle d s \geq 0, \quad \text { a.e., a.s, }
\end{aligned}
$$

where $(m(\cdot), n(\cdot, \cdot), p(\cdot))$ is the solution of the adjoint equation (68).

Remark 11. When the terminal condition $\psi(\cdot)$ is replaced by $\psi(\cdot)+\varphi(X(T))$ in (3), the above methods can still go through.

\section{Examples}

First we will give an example associated with the model studied above. 
Example 1. Consider the following controlled system $(m=$ $d=1)$ :

$$
\begin{gathered}
X(t)=\int_{0}^{t} t u(s) d B_{s} \\
Y(t)=\psi(t)+\int_{t}^{1}(t-1) u(s) d s \\
-\int_{t}^{1} Z(t, s) d B_{s}, \quad t \in[0,1],
\end{gathered}
$$

with the control domain

$$
\begin{gathered}
\mathcal{U}=\left\{(\psi(\cdot), u(\cdot)) \mid \psi(\cdot) \in L_{\mathscr{F}_{T}}^{2}(0,1),\right. \\
u(\cdot) \in L_{\mathbb{F}}^{2}(0,1), \psi(t) \in[0,1], \\
\left.u(t) \in\left[-\frac{1}{2}, 1\right], \text { a.e., a.s. }\right\}
\end{gathered}
$$

and the objective function

$$
J(\psi(\cdot), u(\cdot))=E\left\{X(1)^{2}+Y(0)\right\} .
$$

We will minimize the objective function under the constraints $(\psi(\cdot), u(\cdot)) \in \mathcal{U}$. After substituting $X(1), Y(0)$ into the objective function, we get

$$
J(\psi(\cdot), u(\cdot))=E\left[\int_{0}^{1} u(s)^{2} d s+\psi(0)-\int_{0}^{1} u(s) d s\right] .
$$

From (74), we obtain the optimal control:

$$
\begin{aligned}
& \psi^{*}(s)= \begin{cases}0, & s=0, \\
\text { values in }[0,1], & s \in(0,1],\end{cases} \\
& u^{*}(s)=\frac{1}{2}, \quad s \in[0,1] .
\end{aligned}
$$

So, $\min _{(\psi(\cdot), u(\cdot)) \in \mathscr{U}} J(\psi(\cdot), u(\cdot))=-(1 / 4)$.

At last, we give an example to show the form of the optimal terminal $\psi(\cdot)$.

Example 2. For convenience, we suppose that $m=d=1$, and we consider a simple BSVIE as follows:

$$
\begin{aligned}
Y(t)= & \psi(t)+\int_{t}^{1}[A Y(s)+B Z(s, t)] d s \\
& -\int_{t}^{1} Z(t, s) d B_{s}, \quad t \in[0,1],
\end{aligned}
$$

$A, B \in \mathbb{R}$. We will maximize the objective function $J(\psi(\cdot))=$ $(1 / 2) E\left[\int_{0}^{1} \psi(s)^{2} d s\right]$, subject to $\psi(\cdot) \in L_{\mathscr{F}_{T}}^{2}(0,1), \psi(t) \in[0,1]$, $E Y^{\psi}(t)=\rho(t), t \in[0,1]$, a.e., a.s.

From Section 3.4, we know that the adjoint process $p(\cdot)$ satisfies

$$
\begin{aligned}
p(t)= & h_{0}(t)+(A+B) \bar{h}_{0}+\int_{0}^{T} A p(s) d s \\
& +\int_{0}^{t} B p(s) d B_{s}, \quad t \in[0,1] .
\end{aligned}
$$

Applying Theorem 9, we have that, if $\psi^{*}(\cdot)$ is optimal to $J(\psi(\cdot))$, there exists a deterministic function $h_{0}(\cdot)$, and $\bar{h}_{1} \leq 0$, $\left|h_{0}(\cdot)\right|+\left|\bar{h}_{1}\right| \neq 0$ such that, for any $\psi(\cdot)$,

$$
\left(p(t)+\bar{h}_{1} \psi^{*}(t)\right)\left(\psi(t)-\psi^{*}(t)\right) \geq 0, \quad t \in[0,1], \text { a.s. }
$$

Similar to the example in Ji and Zhou [33], letting $\Omega_{1}:=$ $\left\{(\omega, t) \in \Omega \times[0,1] \mid \psi^{*}(t, \omega)=0\right\}, \Omega_{2}:=\{(\omega, t) \in \Omega \times[0,1] \mid$ $\left.\psi^{*}(t, \omega)=1\right\}$, we obtain that $\psi^{*}(\cdot)$ satisfies

$$
\begin{gathered}
p(t)+\bar{h}_{1} \psi^{*}(t) \geq 0, \quad(\omega, t) \in \Omega_{1}, \text { a.s. } \\
p(t)+\bar{h}_{1} \psi^{*}(t) \leq 0, \quad(\omega, t) \in \Omega_{2}, \text { a.s. } \\
p(t)+\bar{h}_{1} \psi^{*}(t)=0, \quad(\omega, t) \in \Omega-\Omega_{1}-\Omega_{2}, \text { a.s. }
\end{gathered}
$$

\section{Conflict of Interests}

The authors declare that there is no conflict of interests regarding the publication of this paper.

\section{Acknowledgments}

The authors thank Professor Jiongmin Yong and Dr. Tianxiao Wang for their helpful suggestions and discussions. This work was supported by the National Natural Science Foundation of China (nos. 11171187, 11222110, and 11221061). This work was supported by Shandong Province (no. JQ201202), by the Program for New Century Excellent Talents in University of China, and by Postdoctoral Scientific Research Project of Jilin Province (no. RB201357).

\section{References}

[1] M. I. Kamien and E. Muller, "Optimal control with integral state equations," The Review of Economic Studies, vol. 43, no. 3, pp. 469-473, 1976.

[2] M. Berger and V. Mizel, "Volterra equations with Ito integralsI," Journal of Integral Equations and Applications, vol. 2, pp. 187245, 1980.

[3] M. Berger and V. Mizel, "Volterra equations with Ito integralsII," Journal of Integral Equations and Applications, vol. 2, pp. 319337,1980

[4] C. Doléans-Dade, "On the existence and unicity of solutions of stochastic integral equations," Zeitschrift für Wahrscheinlichkeitstheorie und Verwandte Gebiete, vol. 36, no. 2, pp. 93-101, 1976.

[5] A. M. Kolodiu, "Existence of solutions of stochastic Volterra integral equations," Theory of Random Processes, vol. 11, pp. 5157, 1983 (Russian).

[6] E. Pardoux and P. Protter, "Stochastic Volterra equations with anticipating coefficients," The Annals of Probability, vol. 18, no. 4, pp. 1635-1655, 1990.

[7] P. Protter, "Volterra equations driven by semimartingales," The Annals of Probability, vol. 13, no. 2, pp. 519-530, 1985.

[8] T. X. Wang, " $L^{p}$ solutions of backward stochastic Volterra integral equations," Acta Mathematica Sinica, vol. 28, no. 9, pp. 1875-1882, 2012. 
[9] T. Wang and Y. Shi, "A class of time inconsistent risk measures and backward stochastic Volterra integral equations," Risk and Decision Analysis, vol. 4, no. 1, pp. 17-24, 2013.

[10] Y. Shi and T. Wang, "Solvability of general backward stochastic Volterra integral equations," Journal of the Korean Mathematical Society, vol. 49, no. 6, pp. 1301-1321, 2012.

[11] T. Wang and Y. Shi, "Symmetrical solutions of backward stochastic Volterra integral equations and their applications," Discrete and Continuous Dynamical Systems B, vol. 14, no. 1, pp. 251-274, 2010.

[12] Y. Shi, T. Wang, and J. Yong, "Mean-field backward stochastic Volterra integral equations," Discrete and Continuous Dynamical Systems B, vol. 18, no. 7, pp. 1929-1967, 2013.

[13] T. Wang, Q. Zhu, and Y. Shi, "Necessary and sufficient conditions of optimality for stochastic integral systems with partial information," in Proceedings of the 30th Chinese Control Conference (CCC '11), pp. 1950-1955, July 2011.

[14] J. Yong, "Backward stochastic Volterra integral equations and some related problems," Stochastic Processes and their Applications, vol. 116, no. 5, pp. 779-795, 2006.

[15] J. Lin, "Adapted solution of a backward stochastic nonlinear Volterra integral equation," Stochastic Analysis and Applications, vol. 20, no. 1, pp. 165-183, 2002.

[16] J. Yong, "Well-posedness and regularity of backward stochastic Volterra integral equations," Probability Theory and Related Fields, vol. 142, no. 1-2, pp. 21-77, 2008.

[17] D. Laibson, "Golden eggs and hyperbolic discounting," Quarterly Journal of Economics, vol. 112, no. 2, pp. 443-478, 1997.

[18] R. H. Strotz, "Myopia and inconsistency in dynamic utility maximization," The Review of Economic Studies, vol. 23, no. 3, pp. 165-180, 1956.

[19] D. Duffie and L. G. Epstein, "Stochastic differential utility," Econometrica, vol. 60, no. 2, pp. 353-394, 1992.

[20] P. Artzner, F. Delbaen, J.-M. Eber, and D. Heath, "Coherent measures of risk," Mathematical Finance, vol. 9, no. 3, pp. 203-228, 1999.

[21] P. Cheridito, F. Delbaen, and M. Kupper, "Coherent and convex monetary risk measures for bounded càdlàg processes," Stochastic Processes and their Applications, vol. 112, no. 1, pp. 1-22, 2004.

[22] S. Peng, "Nonlinear expectations, nonlinear evaluations and risk measures," in Stochastic Methods in Finance, vol. 1856 of Lecture Notes in Mathematics, pp. 165-253, Springer, Berlin, Germany, 2004.

[23] T. Wang, A Class of Dynamic Risk Measures, University of British Columbia, British Columbia, Canada, 2002, http:// finance.commerce.ubc.ca/research/abstracts/UBCFIN98-5.html.

[24] I. Ekeland and A. Lazrak, "Being serious about noncommitment: subgame perfect equilibrium in continuous time," submitted, 2006, http://arxiv.org/abs/math/0604264vl.

[25] I. Ekeland and T. A. Pirvu, "Investment and consumption without commitment," Mathematics and Financial Economics, vol. 2, no. 1, pp. 57-86, 2008.

[26] J. Yong, "A deterministic linear quadratic time-inconsistent optimal control problem," Mathematical Control and Related Fields, vol. 1, no. 1, pp. 83-118, 2011.

[27] J. Yong, "A deterministic linear quadratic time-inconsistent optimal control problem," Mathematical Control and Related Fields, vol. 1, no. 1, pp. 83-118, 2011.

[28] J. Yong, "Continuous-time dynamic risk measures by backward stochastic Volterra integral equations," Applicable Analysis. An International Journal, vol. 86, no. 11, pp. 1429-1442, 2007.
[29] T. Wang and Y. Shi, "A maximum principle for forwardbackward stochastic Volterra integral equations and applications in finance," submitted, 2010, http://arxiv.org/abs/1004 .2206 .

[30] S. Pliska, Introduction to Mathematical Finance, Blackwell, 1997.

[31] N. El Karoui, S. Peng, and M. C. Quenez, "A dynamic maximum principle for the optimization of recursive utilities under constraints," The Annals of Applied Probability, vol. 11, no. 3, pp. 664-693, 2001.

[32] S. Ji and S. Peng, “Terminal perturbation method for the backward approach to continuous time mean-variance portfolio selection," Stochastic Processes and their Applications, vol. 118, no. 6, pp. 952-967, 2008.

[33] S. Ji and X. Y. Zhou, "A maximum principle for stochastic optimal control with terminal state constraints, and its applications," Communications in Information and Systems, vol. 6, no. 4, pp. 321-338, 2006.

[34] S. Ji and X. Y. Zhou, "A generalized Neyman-Pearson lemma for $g$-probabilities," Probability Theory and Related Fields, vol. 148, no. 3-4, pp. 645-669, 2010.

[35] C. Bernard, S. Ji, and W. Tian, "An optimal insurance design problem under Knightian uncertainty," Decisions in Economics and Finance, vol. 36, no. 2, pp. 99-124, 2013.

[36] S. Ji, "Dual method for continuous-time Markowitz's problems with nonlinear wealth equations," Journal of Mathematical Analysis and Applications, vol. 366, no. 1, pp. 90-100, 2010.

[37] S. L. Ji and Q. M. Wei, "An overview on the principal-agent problems in continuous time," in Real Options, Ambiguity, Risk and Insurance, A. Bensoussan, S. Peng, and J. Sung, Eds., vol. 5 of Studies in Probability, Optimization and Statistics, IOS Press, 2013.

[38] S. Ji and Q. Wei, "A maximum principle for fully coupled forward-backward stochastic control systems with terminal state constraints," Journal of Mathematical Analysis and Applications, vol. 407, no. 2, pp. 200-210, 2013.

[39] S. Ji, Q. Wei, and X. Zhang, "A maximum principle for controlled time-symmetric forward-backward doubly stochastic differential equation with initial-terminal sate constraints," Abstract and Applied Analysis, vol. 2012, Article ID 537376, 29 pages, 2012.

[40] S. L. Ji and Z. Wu, "The maximum principle for one kind of stochastic optimization problem and application in dynamic measure of risk," Acta Mathematica Sinica, vol. 23, no. 12, pp. 2189-2204, 2007.

[41] S. Ji and X. Y. Zhou, "The Neyman-Pearson lemma under $g$ probability," Comptes Rendus Mathématique I, vol. 346, no. 3-4, pp. 209-212, 2008.

[42] L. Epstein and S. L. Ji, "Ambiguous volatility and asset pricing in continuous time," The Review of Financial Studies, vol. 26, no. 7, pp. 1740-1786, 2013.

[43] L. Epstein and S. L. Ji, "Ambiguous volatility, possibility and utility in continuous time," Journal of Mathematical Economics, 2013.

[44] I. Ekeland, "On the variational principle," Journal of Mathematical Analysis and Applications, vol. 47, pp. 324-353, 1974. 


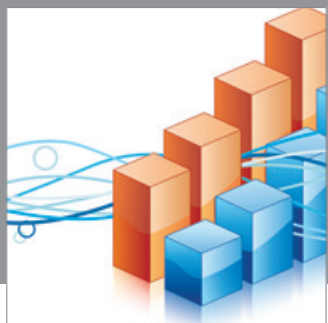

Advances in

Operations Research

mansans

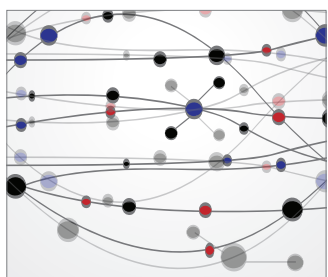

The Scientific World Journal
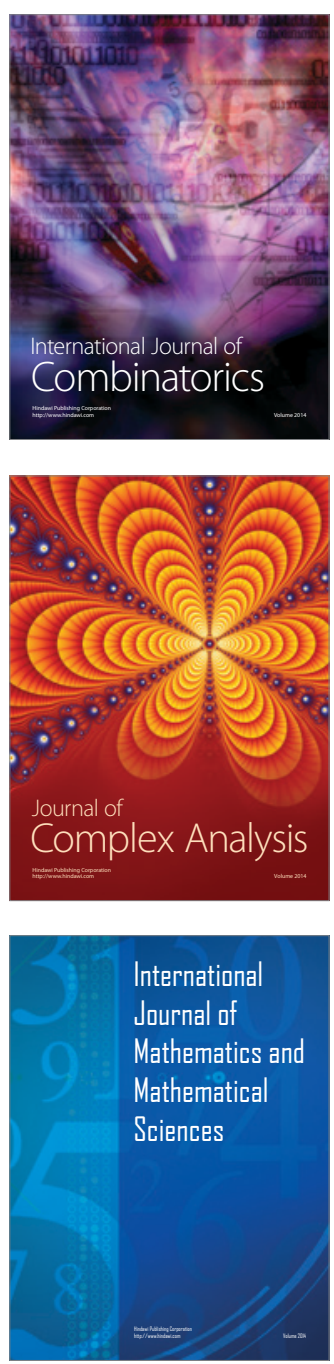
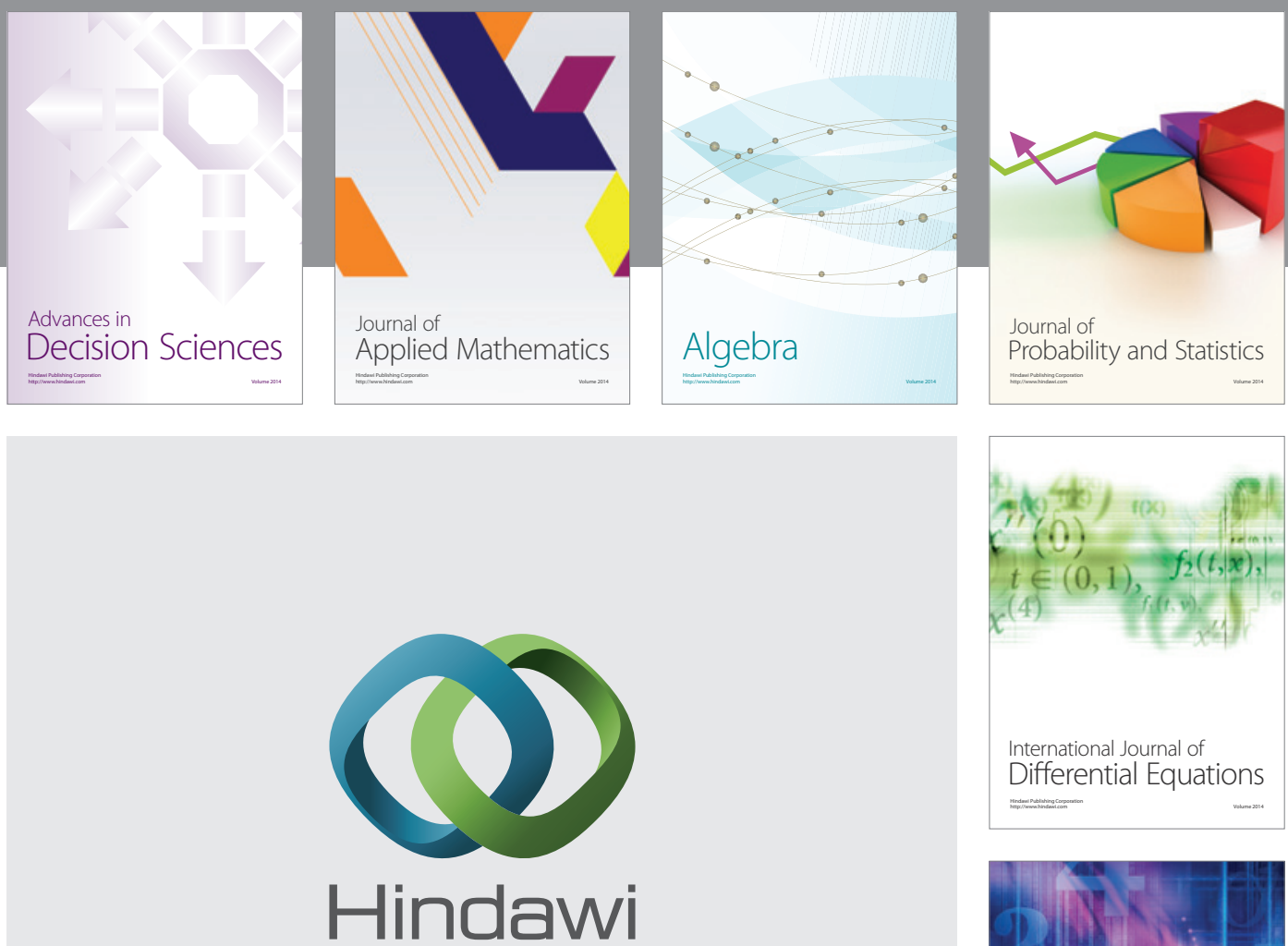

Submit your manuscripts at http://www.hindawi.com
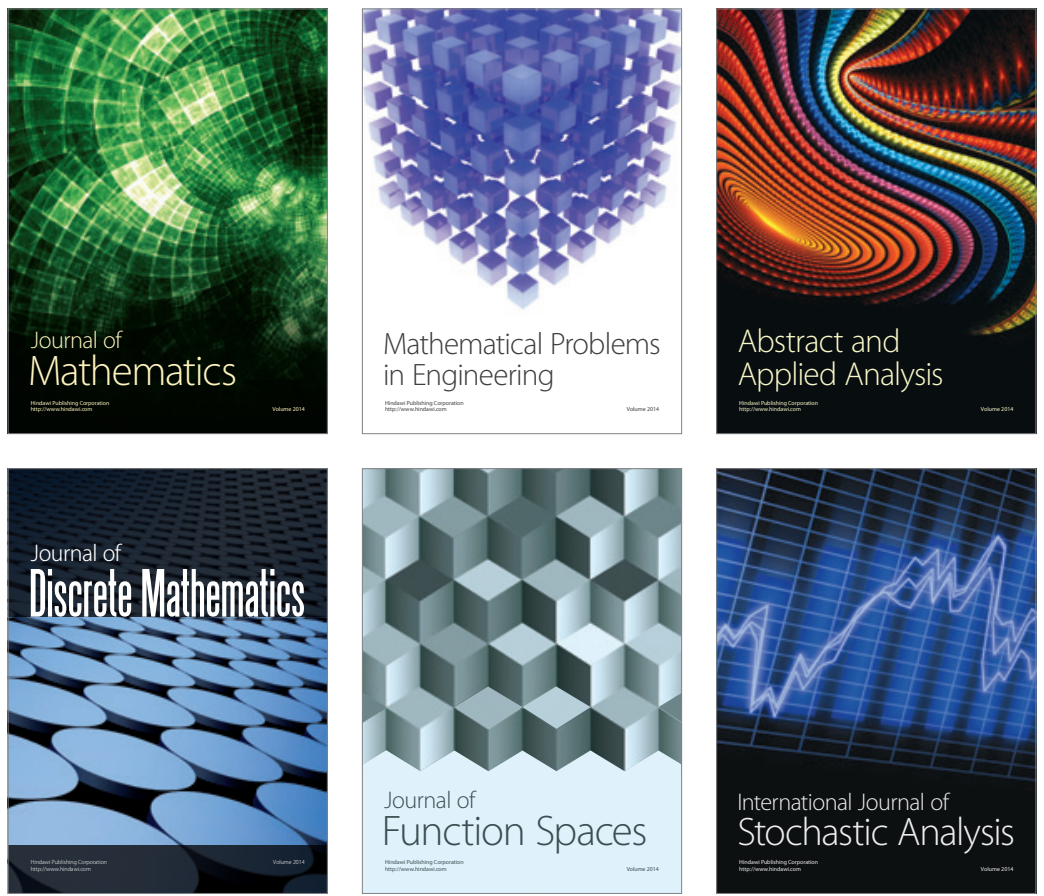

Journal of

Function Spaces

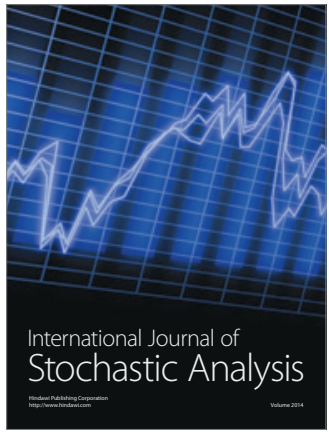

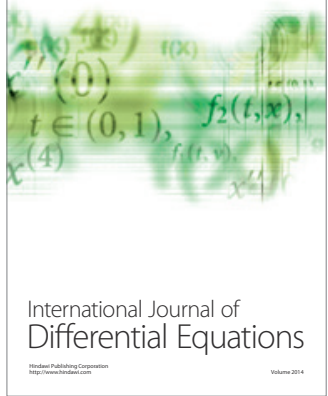
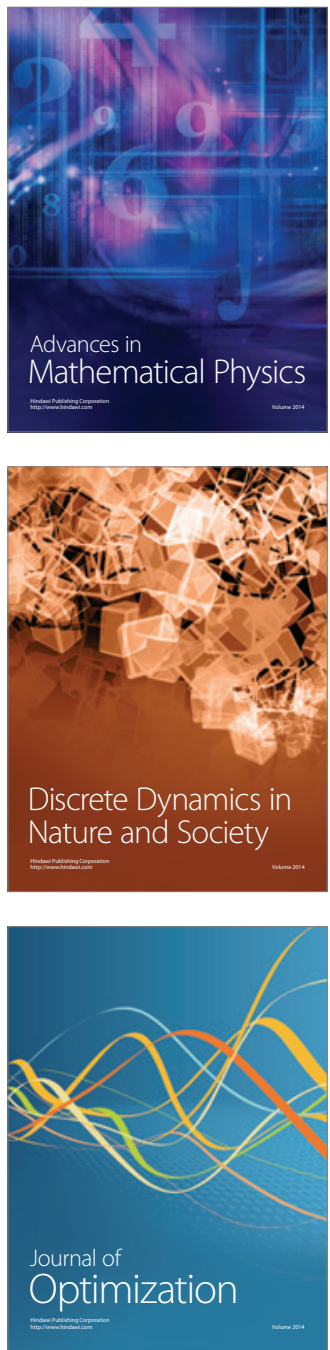